\title{
MEASURING GALAXY STAR FORMATION RATES FROM INTEGRATED PHOTOMETRY: INSIGHTS FROM COLOR-MAGNITUDE DIAGRAMS OF RESOLVED STARS
}

\author{
Benjamin D. Johnson ${ }^{1}$, Daniel R. Weisz ${ }^{2}$, Julianne J. Dalcanton ${ }^{2}$, L. C. Johnson ${ }^{2}$, Daniel A. Dale ${ }^{3}$, \\ Andrew E. Dolphin ${ }^{4}$, Armando Gil de Paz ${ }^{5}$, Robert C. Kennicutt, Jr. ${ }^{6}, \mathrm{Jani}_{\mathrm{A}}$ C. LeE $^{7}$, \\ Evan D. Skillman ${ }^{8}$, MÈdèric Boquien ${ }^{9}$, and Benjamin F. Williams ${ }^{2}$ \\ ${ }^{1}$ Institute d'Astrophysique de Paris, CNRS, UPMC, 98bis Bd Arago, F-75014 Paris, France \\ ${ }^{2}$ Department of Astronomy, Box 351580, University of Washington, Seattle, WA 98195, USA \\ ${ }^{3}$ Department of Physics and Astronomy, University of Wyoming, Laramie, WY 82071, USA \\ ${ }^{4}$ Raytheon, 1151 E. Hermans Road, Tucson, AZ 85756, USA \\ ${ }^{5}$ CEI Campus Moncloa, UCM-UPM, Departamento de Astrofísica y CC. de la Atmósfera, Facultad de CC. Físicas, \\ Universidad Complutense de Madrid, Avda. Complutense s/n, E-28040 Madrid, Spain \\ ${ }^{6}$ Institute of Astronomy, University of Cambridge, Madingley Road, Cambridge CB3 OHA, UK \\ ${ }^{7}$ Space Telescope Science Institute, 3700 San Martin Drive, Baltimore, MD 21218, USA \\ ${ }^{8}$ Department of Astronomy, University of Minnesota, 116 Church Street SE, Minneapolis, MN 55455, USA \\ ${ }^{9}$ Marseille Universitè, CNRS, LAM (Laboratoire d'Astrophysique de Marseille) UMR 7326, F-13388 Marseille, France \\ Received 2012 August 9; accepted 2013 May 15; published 2013 June 27
}

\begin{abstract}
We use empirical star formation histories (SFHs), measured from Hubble-Space-Telescope-based resolved star color-magnitude diagrams, as input into population synthesis codes to model the broadband spectral energy distributions (SEDs) of 50 nearby dwarf galaxies $\left(6.5<\log M_{*} / M_{\odot}<8.5\right.$, with metallicities $\sim 10 \%$ solar). In the presence of realistic SFHs, we compare the modeled and observed SEDs from the ultraviolet (UV) through near-infrared and assess the reliability of widely used UV-based star formation rate (SFR) indicators. In the FUV through $i$ bands, we find that the observed and modeled SEDs are in excellent agreement. In the Spitzer $3.6 \mu \mathrm{m}$ and $4.5 \mu \mathrm{m}$ bands, we find that modeled SEDs systematically overpredict observed luminosities by up to $\sim 0.2$ dex, depending on treatment of the TP-AGB stars in the synthesis models. We assess the reliability of UV luminosity as a SFR indicator, in light of independently constrained SFHs. We find that fluctuations in the SFHs alone can cause factor of $\sim 2$ variations in the UV luminosities relative to the assumption of a constant SFH over the past $100 \mathrm{Myr}$. These variations are not strongly correlated with UV-optical colors, implying that correcting UV-based SFRs for the effects of realistic SFHs is difficult using only the broadband SED. Additionally, for this diverse sample of galaxies, we find that stars older than $100 \mathrm{Myr}$ can contribute from $<5 \%-100 \%$ of the present day UV luminosity, highlighting the challenges in defining a characteristic star formation timescale associated with UV emission. We do find a relationship between UV emission timescale and broadband UV-optical color, though it is different than predictions based on exponentially declining SFH models. Our findings have significant implications for the comparison of UV-based SFRs across low-metallicity populations with diverse SFHs.
\end{abstract}

Key words: galaxies: dwarf - galaxies: fundamental parameters - galaxies: photometry - galaxies: star formation - galaxies: stellar content

Online-only material: color figures

\section{INTRODUCTION}

Measuring the stellar mass and star formation rate (SFR) from a galaxy's observed spectral energy distribution (SED) relies on stellar population synthesis (SPS) models. These models combine knowledge of stellar evolution and stellar spectra to convert between observations and physical quantities. In recent years it has become common to compare observed galaxies to model SEDs across a range of wavelengths to derive multiple galaxy properties (e.g., stellar mass, SFR, metallicity) selfconsistently. Fitting model SEDs to observed SEDs is now done for both low and high redshift galaxies (e.g., Arnouts et al. 2007; Salim et al. 2007; Schaerer \& de Barros 2010; Whitaker et al. 2012; Curtis-Lake et al. 2013; Maraston et al. 2012; Mentuch Cooper et al. 2012), and from ultraviolet (UV) to far-infrared (FIR) wavelengths (e.g., Silva et al. 1998; da Cunha et al. 2008; Noll et al. 2009).

Star formation histories (SFHs) are a critical component of SED modeling. A different SFH can change the relationship between physical quantities and the SED. However, the SFH of individual galaxies is usually poorly or only coarsely known, and some assumption about its form must then be made. The simplest models assume a constant SFR to derive linear scalings between SFR and luminosity (e.g., Kennicutt 1998). More sophisticated modeling involves allowing the SFR to vary with time, though it is usually parameterized to be a smoothly varying function. A common parameterization is the $\tau$-model, where the SFR declines exponentially (Tinsley 1968; Madau et al. 1998; Kauffmann et al. 2003; Walcher et al. 2011). The timescale and amplitude of this parameterized SFH is then constrained by the SED itself.

Difficulties in this approach arise from (1) well-known and significant degeneracies between SFH, dust attenuation, and stellar metallicity (e.g., Johnson et al. 2007a; Walcher et al. 2011 and references therein); and (2) biases in the derived parameters due to true SFHs that deviate from the assumed parameterization (e.g., with a different long-term SFR evolution or variable SFR on short timescales). Recently, Lee et al. (2010) have shown, using SFHs drawn from semi-analytic models, that determinations of the physical parameters of high-redshift 
galaxies can be significantly biased if they are derived from fitting SED models that assume a simplified or mismatched SFH (see also Stringer et al. 2011; Pforr et al. 2012). These results depend on the adopted semi-analytic model of the SFH, and are valid at high-redshift. How well they apply to real galaxies in the low-redshift universe is unknown (though see Wilkins et al. 2012).

Instead of assuming a parameterized SFH, strong constraints on the real SFH can be obtained from a galaxy's resolved stellar populations (e.g., Tosi et al. 1989; Dolphin 2002). The location of individual stars in a color-magnitude diagram (CMD) constrains their evolutionary state, and can be used to infer the SFH for an assumed initial mass function (IMF). Studies of the resolved stellar populations of nearby galaxies are now routine with Hubble Space Telescope (HST; e.g., Sanna et al. 2009; Dalcanton et al. 2009; Hidalgo et al. 2011; Grocholski et al. 2012; Dalcanton et al. 2012). With the SFH thus constrained by the CMD of resolved stars, we can determine the impact of realistic SFHs on typical conversions between observed and physical properties.

One advantage of using the SFH measured for real galaxies, as opposed to a SFH drawn from semi-analytic models, is that it is possible to directly compare the measured SED to the SED inferred from the SFH. This comparison allows us to test the consistency of the SFH and population synthesis models with broadband observations from the ultraviolet (UV) to the nearinfrared (NIR). Another advantage of using the SFH measured for real galaxies is that we can explore the effects of other galaxy properties on the SED (e.g., reddening by dust). The realistic $\mathrm{SFH}$ inferred from the CMD of individual stars serves to fix a large number of parameters in the SED model that usually have to be fit at the same time as other, possibly degenerate, parameters.

The paper is organized as follows. In Section 2 we describe the sample of galaxies, the broadband UV through MIR observations, the SFHs as derived from the resolved stellar CMDs of each galaxy, and how we predict the broadband SED using the CMD-constrained SFHs as input. In Section 3 we compare the predicted luminosities to the observed luminosities in every band, highlighting discrepancies in the NIR. In Section 4 we explore the possible origins of these discrepancies, including uncertainties in the population synthesis model ingredients. In Section 5 we consider the effects of dust attenuation, metallicity, and stochastic sampling of the IMF on the observed SED. In Section 6 we investigate how the SFH affects the conversion of UV luminosity to SFR.

\section{DATA}

\subsection{Sample}

We analyze galaxies from the ACS Nearby Galaxy Survey Treasury (ANGST; Dalcanton et al. 2009), which consists primarily of dwarf galaxies $\left(M_{*}<10^{9} M_{\odot}\right)$ with distances less than $4 \mathrm{Mpc}$. The sample spans a range of colors, morphologies (dE to $\mathrm{dIm}$ ), and SFHs. We have chosen a subset of ANGST galaxies for which HST observations sample a significant fraction of the total galaxy extent, and where the depth and quality of the observations provide for robust measures of the SFH (Weisz et al. 2011). The 50 selected galaxies are listed in Table 1, along with their distances and other global properties. In Figure 1 we show the specific SFR (the SFR divided by the stellar mass) versus stellar mass (where both quantities were derived from the CMDs, see Sections 2.4 and 2.5)

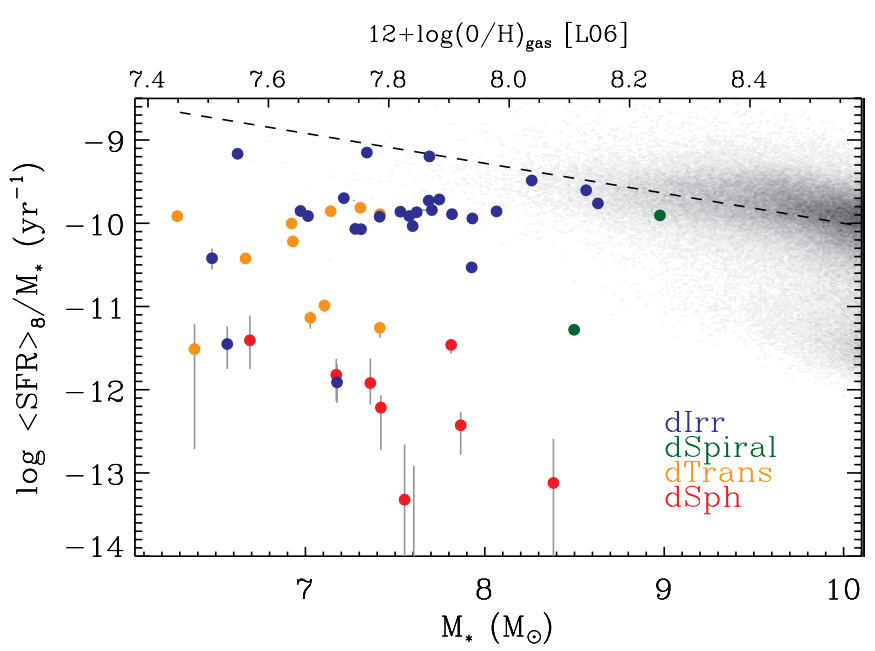

Figure 1. Specific star formation rate vs. stellar mass for the sample galaxies, where both have been derived from the SFH. For the stellar mass, we have applied a correction based on the amount of $3.6 \mu \mathrm{m}$ light falling outside the $H S T$ footprint to obtain an estimate of the total stellar mass. The error bars are derived from the Monte Carlo realizations of the SFH, and encompass $68 \%$ of the distribution of values. For the stellar mass these errors are typically smaller than the symbol size. Each point is color-coded by the morphological type. The top axis shows the gas-phase metallicity inferred from the mass-metallicity relation of Lee et al. (2006). Gray-scale shows galaxies from the SDSS main galaxy sample (Brinchmann et al. 2004). The dashed line is an extrapolation to lower mass of the SF sequence determined by Schiminovich et al. (2007).

(A color version of this figure is available in the online journal.)

for the sample galaxies, color-coded by morphological type (Section 2.3).

\subsection{Broadband SEDs}

The integrated galaxy SEDs are derived from broadband imaging available from GALEX (Martin et al. 2005), the Sloan Digital Sky Survey (SDSS), and Spitzer (Werner et al. 2004). The GALEX and Spitzer imaging has been obtained as part of the 11HUGS and LVL surveys, respectively (Lee et al. 2011; Dale et al. 2009), and we refer the reader to these papers for detailed discussion of the data reduction. Additional optical imaging for galaxies outside the SDSS footprint has been obtained by D. O. Cook et al. (2013, in preparation), through the Johnson-Cousins $U B V R$ filters. Unfortunately, the low surface brightness of these galaxies makes NIR $J, H$, and $K$ measurements difficult from the ground (e.g., McIntosh et al. 2006), and we do not consider these bands here.

We have measured the broadband luminosity that falls within the intersection between the HST footprint and large apertures designed to encompass the entire UV and NIR extent of the galaxy as follows. For the SDSS imaging we estimate the background from Gaussian fits to the lower $85 \%$ of flux values in elliptical annuli extending from 1.5 to $\sim 2$ times the semimajor axis of the galaxy aperture. These estimates are consistent with the SDSS pipeline values outside the galaxy extent (see Blanton et al. 2011 for a discussion of biases in the SDSS background determination near large galaxies). For the Spitzer Infrared Array Camera (IRAC; Fazio et al. 2004) imaging we use the background determinations of Dale et al. (2009). For the GALEX imaging we estimate the background by median filtering the GALEX pipeline-produced background images in the same elliptical annuli as was used for the optical backgrounds. The GALEX pipeline-produced background images include masking of detected sources and account for the Poisson statistics of the 
Table 1

Galaxy Sample Properties

\begin{tabular}{|c|c|c|c|c|c|c|c|c|}
\hline Name & $\begin{array}{l}\text { Alternate } \\
\text { Name }\end{array}$ & $\begin{array}{l}\log \langle\mathrm{SFR}\rangle_{8}^{\mathrm{a}} \\
\left(M_{\odot} \mathrm{yr}^{-1}\right)\end{array}$ & $\begin{array}{c}\log M_{*}^{\mathrm{a}} \\
\left(M_{\odot}\right)\end{array}$ & $\begin{array}{l}A_{\text {fuv }}{ }^{\mathrm{b}} \\
(\mathrm{mag})\end{array}$ & $E(B-V)_{\mathrm{MW}}$ & $\begin{array}{c}\log [\mathrm{O} / \mathrm{H}]+12^{\mathrm{c}} \\
(\mathrm{B} 12)\end{array}$ & $f_{\mathrm{HST}^{\mathrm{d}}}^{\mathrm{d}}$ & $\overline{T \text { Type }}$ \\
\hline AM1001-270 & Antlia & $-4.086_{-0.116}^{+0.400}$ & $6.666_{-0.021}^{+0.025}$ & $<0.34$ & 0.079 & 7.41 & 1.00 & 10 \\
\hline BK5N & $\ldots$ & $-4.706_{-0.203}^{+0.372}$ & $7.293_{-0.024}^{+0.009}$ & $<4.78$ & 0.063 & 7.55 & 1.00 & -3 \\
\hline UGCA276 & DDO113 & $-4.788_{-0.270}^{+0.216}$ & $7.129_{-0.013}^{+0.008}$ & $<0.12$ & 0.020 & 7.57 & 1.00 & 10 \\
\hline UGC7577 & DDO125 & $-2.171_{-0.019}^{+0.028}$ & $7.775_{-0.013}^{+0.016}$ & $0.04 \pm 0.006$ & 0.020 & 7.78 & 0.77 & 10 \\
\hline UGC8651 & DDO181 & $-2.456_{-0.018}^{+0.018}$ & $7.580_{-0.010}^{+0.003}$ & $0.04 \pm 0.006$ & 0.006 & 7.67 & 0.97 & 10 \\
\hline UGC8760 & DDO183 & $-2.349_{-0.014}^{+0.016}$ & $7.567_{-0.011}^{+0.011}$ & $0.02 \pm 0.003$ & 0.016 & 7.70 & 1.00 & 10 \\
\hline UGC9128 & DDO187 & $-2.880_{-0.023}^{+0.034}$ & $7.039_{-0.007}^{+0.006}$ & $<0.11$ & 0.023 & 7.56 & 1.00 & 10 \\
\hline UGC9240 & DDO190 & $-2.102_{-0.009}^{+0.013}$ & $7.791_{-0.011}^{+0.005}$ & $0.14 \pm 0.015$ & 0.012 & 7.77 & 0.96 & 10 \\
\hline UGCA133 & DDO044 & $-4.795_{-0.505}^{+0.148}$ & $7.420_{-0.004}^{+0.009}$ & $<4.92$ & 0.041 & 7.65 & 1.00 & -3 \\
\hline UGCA015 & DDO006 & $-2.460_{-0.014}^{+0.007}$ & $7.351_{-0.005}^{+0.009}$ & $<0.17$ & 0.017 & 7.60 & 1.00 & 10 \\
\hline DDO078 & $\ldots$ & $-4.676_{-0.316}^{+0.191}$ & $7.782_{-0.012}^{+0.009}$ & $<3.62$ & 0.021 & 7.49 & 0.41 & -3 \\
\hline UGC5692 & DDO082 & $-2.876_{-0.037}^{+0.039}$ & $8.404_{-0.003}^{+0.009}$ & $0.26 \pm 0.032$ & 0.041 & 7.90 & 0.67 & 9 \\
\hline UGC6817 & DDO099 & $-2.288_{-0.030}^{+0.018}$ & $7.565_{-0.059}^{+0.010}$ & $0.05 \pm 0.007$ & 0.026 & 7.67 & 0.74 & 10 \\
\hline ESO294-G010 & $\ldots$ & $-4.118_{-0.126}^{+0.081}$ & $7.024_{-0.005}^{+0.003}$ & $<0.55$ & 0.006 & 7.56 & 0.96 & -3 \\
\hline ESO410-G005 & $\ldots$ & $-3.901_{-0.057}^{+0.089}$ & $7.106_{-0.004}^{+0.005}$ & $<0.55$ & 0.014 & 7.59 & 1.00 & -1 \\
\hline ESO540-G032 & $\ldots$ & $-3.864_{-0.095}^{+0.091}$ & $7.415_{-0.006}^{+0.009}$ & $<1.14$ & 0.020 & 7.52 & 1.00 & -3 \\
\hline F08D1 & $\ldots$ & -4.573 & $8.007_{-0.003}^{+0.012}$ & $<0.92$ & 0.108 & 7.80 & 1.00 & -3 \\
\hline UGC8091 & GR8 & $-2.887_{-0.030}^{+0.032}$ & $6.966_{-0.024}^{+0.020}$ & $0.04 \pm 0.005$ & 0.026 & 7.54 & 1.00 & 10 \\
\hline HS117 & HS98-117 & $-5.017_{-0.201}^{+0.332}$ & $6.548_{-0.014}^{+0.005}$ & $<2.65$ & 0.115 & 7.50 & 0.97 & 10 \\
\hline UGC5666 & IC2574 & $-1.084_{-0.003}^{+0.003}$ & $8.821_{-0.002}^{+0.002}$ & $0.14 \pm 0.016$ & 0.036 & 8.09 & 0.74 & 9 \\
\hline IKN & $\ldots$ & $-3.643_{-0.111}^{+0.049}$ & $7.812_{-0.016}^{+0.011}$ & $<4.67$ & 0.058 & 7.51 & 1.00 & -3 \\
\hline M81-DwA & KDG52 & $-2.715_{-0.014}^{+0.021}$ & $7.146_{-0.008}^{+0.005}$ & $0.20 \pm 0.029$ & 0.020 & 7.48 & 1.00 & 10 \\
\hline KDG61 & KK98-81 & $-3.995_{-0.100}^{+0.102}$ & $7.675_{-0.012}^{+0.010}$ & $<3.28$ & 0.073 & 7.69 & 0.99 & -1 \\
\hline UGC5442 & KDG64 & -5.367 & $7.563_{-0.008}^{+0.005}$ & $1.77 \pm 1.717$ & 0.053 & 7.71 & 1.00 & -3 \\
\hline KDG73 & $\ldots$ & $-3.071_{-0.028}^{+0.025}$ & $6.923_{-0.012}^{+0.025}$ & $<0.83$ & 0.019 & 7.52 & 1.00 & 10 \\
\hline KKR03 & KK98-230 & $-3.697_{-0.060}^{+0.064}$ & $6.222_{-0.011}^{+0.014}$ & $<0.37$ & 0.014 & 7.31 & 1.00 & 10 \\
\hline КKH037 & HS98-010 & $-3.255_{-0.040}^{+0.042}$ & $7.376_{-0.012}^{+0.010}$ & $<0.49$ & 0.074 & 7.60 & 1.00 & 10 \\
\hline KKH086 & $\ldots$ & $-3.927_{-0.099}^{+0.160}$ & $6.525_{-0.023}^{+0.038}$ & $<1.05$ & 0.027 & 7.45 & 1.00 & 10 \\
\hline ККH098 & $\ldots$ & $-3.298_{-0.026}^{+0.052}$ & $6.930_{-0.027}^{+0.018}$ & $<0.22$ & 0.123 & 7.52 & 1.00 & 10 \\
\hline KKR25 & $\ldots$ & $-5.267_{-1.142}^{+0.352}$ & $6.322_{-0.033}^{+0.025}$ & $<4.54$ & 0.009 & 7.29 & 0.89 & 10 \\
\hline NGC 2366 & UGC3851 & $-1.176_{-0.004}^{+0.003}$ & $8.585_{-0.002}^{+0.002}$ & $0.40 \pm 0.039$ & 0.036 & 7.96 & 0.87 & 10 \\
\hline NGC 3741 & UGC6572 & $-2.349_{-0.014}^{+0.019}$ & $7.517_{-0.009}^{+0.006}$ & $0.05 \pm 0.006$ & 0.024 & 7.67 & 0.98 & 10 \\
\hline NGC 4163 & UGC7199 & $-2.615_{-0.015}^{+0.022}$ & $7.927_{-0.007}^{+0.002}$ & $0.12 \pm 0.014$ & 0.020 & 7.79 & 1.00 & 10 \\
\hline UGC4483 & $\ldots$ & $-2.490_{-0.027}^{+0.021}$ & $7.214_{-0.017}^{+0.007}$ & $0.10 \pm 0.011$ & 0.034 & 7.53 & 1.00 & 10 \\
\hline UGC8201 & DDO165 & $-1.220_{-0.005}^{+0.004}$ & $8.267_{-0.030}^{+0.011}$ & $0.02 \pm 0.004$ & 0.024 & 7.86 & 1.00 & 10 \\
\hline UGC8508 & IZw60 & $-2.546_{-0.015}^{+0.016}$ & $7.377_{-0.004}^{+0.003}$ & $<0.00$ & 0.015 & 7.67 & 0.94 & 10 \\
\hline UGC8833 & $\ldots$ & $-2.779_{-0.025}^{+0.024}$ & $7.294_{-0.016}^{+0.010}$ & $<0.16$ & 0.012 & 7.61 & 1.00 & 10 \\
\hline UGCA292 & $\ldots$ & $-2.486_{-0.016}^{+0.030}$ & $6.688_{-0.031}^{+0.022}$ & $0.06 \pm 0.010$ & 0.016 & 7.49 & 1.00 & 10 \\
\hline UGCA438 & ESO407-G018 & $-2.582_{-0.020}^{+0.030}$ & $7.306_{-0.011}^{+0.052}$ & $<0.07$ & 0.014 & 7.66 & 1.00 & 10 \\
\hline UGC5428 & DDO071 & -5.125 & $7.535_{-0.007}^{+0.003}$ & $<0.00$ & 0.098 & 7.66 & 0.98 & -3 \\
\hline Sex A & DDO075 & $-2.370_{-0.010}^{+0.009}$ & $6.793_{-0.015}^{+0.006}$ & $0.03 \pm 0.003$ & 0.045 & 7.70 & 0.31 & 10 \\
\hline ESO321-G014 & $\ldots$ & $-2.800_{-0.034}^{+0.047}$ & $7.286_{-0.016}^{+0.011}$ & $0.01 \pm 0.011$ & 0.094 & 7.67 & 0.96 & 10 \\
\hline ESO540-G030 & KDG02 & $-4.764_{-0.305}^{+0.327}$ & $6.690_{-0.020}^{+0.010}$ & $<1.28$ & 0.023 & 7.60 & 1.00 & -1 \\
\hline IC5152 & ESO237-G027 & $-1.962_{-0.013}^{+0.009}$ & $7.746_{-0.007}^{+0.015}$ & $0.14 \pm 0.015$ & 0.025 & 7.95 & 0.39 & 10 \\
\hline Sculptor-dE1 & $\mathrm{Sc} 22$ & $-4.642_{-0.326}^{+0.167}$ & $7.168_{-0.014}^{+0.012}$ & $<5.07$ & 0.015 & 7.50 & 0.99 & -3 \\
\hline UGC5373 & Sex B, DDO070 & $-2.753_{-0.020}^{+0.023}$ & $7.126_{-0.013}^{+0.006}$ & $0.05 \pm 0.006$ & 0.031 & 7.74 & 0.33 & 10 \\
\hline UGC4305 & Ho II, DDO050 & $-1.173_{-0.002}^{+0.003}$ & $8.432_{-0.002}^{+0.001}$ & $0.11 \pm 0.012$ & 0.032 & 8.00 & 0.80 & 10 \\
\hline UGC4459 & DDO053 & $-2.299_{-0.015}^{+0.017}$ & $7.687_{-0.012}^{+0.0010}$ & $0.21 \pm 0.023$ & 0.038 & 7.69 & 1.00 & 10 \\
\hline UGC5139 & Ho I, DDO063 & $-1.799_{-0.010}^{+0.006}$ & $8.059_{-0.006}^{+0.005}$ & $0.04 \pm 0.006$ & 0.050 & 7.78 & 0.97 & 10 \\
\hline UGC5336 & Ho IX, DDO066 & $-1.529_{-0.005}^{+0.007}$ & $7.668_{-0.012}^{+0.010}$ & $0.03 \pm 0.005$ & 0.079 & 7.71 & 0.98 & 10 \\
\hline
\end{tabular}

Notes.

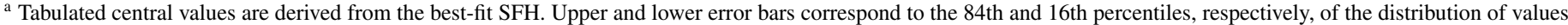

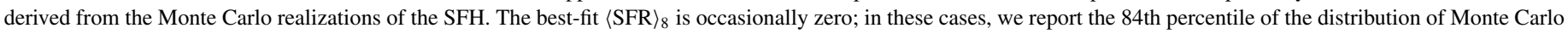
values (an approximate $1 \sigma$ upper limit) and no error bars are given.

${ }^{\mathrm{b}}$ Internal dust attenuation estimated from the ratio of the $24 \mu \mathrm{m}$ to FUV flux following Hao et al. (2011).

${ }^{c}$ Gas-phase metallicity as estimated from the $4.5 \mu \mathrm{m}$ luminosity-metallicity relation of Berg et al. (2012).

${ }^{\mathrm{d}}$ Fraction of the $g$ or $B$ band flux within the Dale et al. (2009) apertures that also falls within the HST FOV, i.e., an aperture correction. 
low count-rate images. We subtract these backgrounds from the flux that falls in the HST footprints and the galaxy apertures.

For GALEX and Spitzer we use the standard photometric zeropoints. For the SDSS data the photometric calibration is taken from the calibration data provided for each SDSS imaging frame. Masking of foreground stars and artifacts follows Dale et al. (2009) and Lee et al. (2011), though we have inspected the foreground masks by hand for these galaxies to be sure that no UV bright clusters are mistakenly masked. For several galaxies, the presence of nearby and extremely bright foreground stars makes accurate photometry impossible. These galaxies are DDO78, KKR025, and IKN. Though we do not consider the photometry at any wavelength for these galaxies, we retain them in the sample since they can still contribute to conclusions based solely on the modeled fluxes (see Sections 2.5 and 6). The quoted photometric uncertainties of the GALEX and Spitzer fluxes are typically dominated by calibration uncertainty (Dale et al. 2009; Lee et al. 2011), but systematics related to sky background estimation (especially in the ground-based $u, U$, and $z$ bands) and unsubtracted foreground stars likely contribute substantially to the true photometric uncertainty.

Our photometry is reported in Table 2 . The fraction of the total $g$ or $B$-band flux that falls within the HST footprint is listed in Table 1. Corrections for Milky Way reddening are derived from the reddening maps of Schlegel et al. (1998); the adopted conversions from $E(B-V)$ to $A_{\lambda}$ are given in Table 3. For the GALEX bands these conversions follow Gil de Paz et al. (2007) and for the optical we derive the conversions from the Milky Way extinction curve of Cardelli et al. (1989) with $R_{V}=3.1$. We convert between absolute magnitude $\left(M_{\lambda_{e}}\right)$ and solar luminosities using

$$
\log v L_{v}=\log c / \lambda_{e}+\left(51.595-M_{\lambda_{e}}\right) / 2.5-\log L_{\odot},
$$

where $\lambda_{e}$ are the effective wavelengths of the bands ${ }^{10}$ as given in Table 3 and $L_{\odot}=3.827 \times 10^{33} \mathrm{erg} \mathrm{s}^{-1}$.

\subsection{Additional Galaxy Properties}

We also consider measures of internal dust attenuation and metallicity, which are known to affect the SED. Accurate estimates of dust attenuation are notoriously difficult to obtain. For the vast majority of the sample, measurements of the Balmer decrement are not available, and so attenuations cannot be measured in this way (Lee et al. 2009). Such measurements also only sample the very youngest stars, not the attenuation of the stellar population as a whole. However, the broad wavelength coverage of this sample allows us to use the infrared-to-UV ratio (IRX), which is available for the majority of the sample. Following Hao et al. (2011), we estimate the FUV attenuation in magnitudes from the ratio of $24 \mu \mathrm{m}$ to FUV flux as

$$
A_{\text {fuv }}=2.5 \log \left(1+\eta \frac{v L_{v}(24 \mu \mathrm{m})}{v L_{v}(\mathrm{FUV})}\right),
$$

where $\eta=3.89$ is a scaling factor determined from observations. This relation has been calibrated by Hao et al. (2011) using attenuations derived from the Balmer decrement, albeit for a sample of galaxies with higher luminosities and larger average dust attenuation. For galaxies undetected at $24 \mu \mathrm{m}$ (24 of the 50 sample galaxies), we use the $1 \sigma 24 \mu \mathrm{m}$ flux limit to define an upper limit on the attenuation. The resulting FUV

\footnotetext{
${ }_{10}$ We use the definition of Schneider et al. (1983) for $\lambda_{e}$.
}

attenuations are given in Table 1 . For the majority of the sample, $A_{\text {fuv }}<0.35 \mathrm{mag}$, consistent with their low luminosities. Therefore, the uncertainties in the method used to derive the attenuation are of minimal importance, because the absolute attenuations are undoubtedly small.

Morphological $T$-types are taken from de Vaucouleurs et al. (1991) and Karachentsev et al. (2004) and are given in Table 1. Following Weisz et al. (2011) we define the galaxy types dwarf spheroidal (dSph, $T \leqslant 0$ ), dwarf irregular (dIrr, $T=10$ ), and dwarf spiral (dSpiral, $7<T<10$ ). We include the dwarf transition (dTrans) type, defined as galaxies with detectable gas content but undetectable $\mathrm{H} \alpha$ emission. The galaxy metallicity may be estimated from a mass-metallicity or luminosity-metallicity relation. We use the $4.5 \mu \mathrm{m}$ luminosity-metallicity relation of Berg et al. (2012) to estimate the gas-phase metallicity. The resulting estimates of the gas-phase metallicity are listed in Table 1 . The metallicities are typically well below solar.

\subsection{Deriving SFHs from Resolved Stars}

We derived SFHs for the sample from their optical stellar CMDs. The methodology involves matching the observed density of stars in color-magnitude space to linear combinations of the CMD density expected from simple stellar populations of various ages, including reddening by dust and observational effects modeled with extensive artificial star tests; a more detailed description can be found in Weisz et al. (2011). The CMD fitting takes into account Poisson statistics when measuring fit quality, and thus implicitly includes the effects of stochasticity in the population of the IMF on the number of stars of a given mass (or luminosity) in the error estimate (see Section 2.6). The constraint on the most recent SFH comes primarily from main sequence stars, whereas the SFH between 50-500 Myr is largely constrained by easily identified helium burning (HeB) stars, which follow a rough luminosity-age relation (DohmPalmer et al. 2002).

The CMDs were derived with stellar metallicity in each age bin as a free parameter, and assuming a Salpeter IMF from 0.1 to $100 M_{\odot}$ (slightly different from the IMF used in Weisz et al. 2011) and the stellar evolutionary tracks of Girardi et al. (2000). Differential extinction of young stars is included following the model of Dolphin et al. (2003). In this model a flat distribution of extinction values is applied to all stars younger than $100 \mathrm{Myr}$, with the maximum extinction increasing linearly from $A_{V}=0.0$ at an age of $100 \mathrm{Myr}$ to $A_{V}=0.5$ mag at an age of 40 Myr. By increasing the width of the main sequence in the simulated CMDs, this differential extinction model gives vastly improved fits to the data over no extinction. In Section 5.3 we discuss the impact of differential reddening and independent estimates of dust content in more detail. The temporal resolution of the derived SFHs is $\Delta \log t=0.1$, which is coarser than the time resolution used for the $\mathrm{SFH}$ derivation in Weisz et al. (2011), though finer than the time resolution displayed in that work. The SFHs are derived within the range $4 \mathrm{Myr}<\log t_{\text {lookback }}<14.1 \mathrm{Gyr}$. An example SFH is shown in Figure 2.

We have used the SFHs to derive $\langle\mathrm{SFR}\rangle_{8}$, the average SFR over the last $100 \mathrm{Myr}$, and $M_{*}$, the current stellar mass, by integrating the SFH over time, accounting for stellar death using population synthesis models (see Section 2.5). The resulting properties are listed in Table 1. The metallicities inferred from the CMD fitting, while uncertain, are consistent with the gasphase metallicities of Section 2.3 and Table 1. 
Table 2

Integrated Photometry within the HST Footprint

\begin{tabular}{|c|c|c|c|c|c|c|c|c|c|c|c|c|}
\hline Name & $\begin{array}{c}(m-M)^{\mathrm{a}} \\
(\mathrm{mag})\end{array}$ & $\begin{array}{l}\text { FUV } \\
\text { (mag) }\end{array}$ & $\begin{array}{l}\text { NUV } \\
\text { (mag) }\end{array}$ & $\begin{array}{l}u(U)^{\mathrm{b}} \\
(\mathrm{mag})\end{array}$ & $\begin{array}{l}g(B)^{\mathrm{b}} \\
(\mathrm{mag})\end{array}$ & $\begin{array}{l}r(V)^{\mathrm{b}} \\
(\mathrm{mag})\end{array}$ & $\begin{array}{l}i(R)^{\mathrm{b}} \\
(\mathrm{mag})\end{array}$ & $\begin{array}{c}z \\
(\mathrm{mag})\end{array}$ & $\begin{array}{c}3.6 \mu \mathrm{m} \\
(\mathrm{mag})\end{array}$ & $\begin{array}{c}4.5 \mu \mathrm{m} \\
(\mathrm{mag})\end{array}$ & $\begin{array}{c}5.6 \mu \mathrm{m} \\
(\mathrm{mag})\end{array}$ & $\begin{array}{l}8 \mu \mathrm{m} \\
(\mathrm{mag})\end{array}$ \\
\hline AM1001-270 & 25.40 & -7.79 & -8.79 & $(-8.26)$ & $(-10.26)$ & $(-10.71)$ & $(-10.77)$ & $\cdots$ & -10.38 & -9.93 & $\cdots$ & -9.67 \\
\hline $\mathrm{BK} 5 \mathrm{~N}$ & 27.89 & -7.89 & -7.56 & $\ldots$ & $\ldots$ & $\ldots$ & $\ldots$ & $\ldots$ & -11.98 & -11.09 & $\ldots$ & -12.56 \\
\hline UGCA276 & 27.35 & -7.08 & -8.92 & $\ldots$ & $\ldots$ & $\ldots$ & $\ldots$ & $\ldots$ & -12.12 & -11.52 & -10.51 & $\ldots$ \\
\hline UGC7577 & 27.02 & -12.01 & -12.35 & -13.31 & -14.12 & -14.46 & -14.62 & -14.70 & -13.78 & -13.19 & -12.58 & -12.17 \\
\hline UGC8651 & 27.40 & -11.62 & -11.83 & -12.55 & -13.32 & -13.58 & -13.71 & -13.87 & -13.02 & -12.52 & -10.87 & -11.14 \\
\hline UGC8760 & 27.51 & -11.57 & -11.90 & -12.58 & -13.38 & -13.59 & -13.82 & -13.74 & -13.13 & -12.75 & -12.31 & $\ldots$ \\
\hline UGC9128 & 26.72 & -10.43 & -10.82 & -11.79 & -12.58 & -12.78 & -12.90 & -12.95 & -12.09 & -11.78 & -10.20 & -11.36 \\
\hline UGC9240 & 27.23 & -12.30 & -12.63 & -13.34 & -14.23 & -14.49 & -14.64 & -14.85 & -13.81 & -13.39 & -13.11 & -13.19 \\
\hline UGCA133 & 27.45 & -6.76 & -9.43 & -11.75 & -12.65 & -13.11 & -13.16 & -13.50 & -12.92 & -12.59 & -10.17 & $\ldots$ \\
\hline UGCA015 & 27.60 & -10.75 & -10.96 & $(-11.78)$ & $(-12.38)$ & $(-12.57)$ & $\ldots$ & $\ldots$ & -12.24 & -11.94 & -10.98 & -10.50 \\
\hline DDO078 & 27.82 & $\ldots$ & $\ldots$ & $\ldots$ & $\ldots$ & $\ldots$ & $\ldots$ & $\ldots$ & $\ldots$ & $\ldots$ & $\ldots$ & $\ldots$ \\
\hline UGC5692 & 28.06 & -11.45 & -12.36 & $(-14.20)$ & $(-14.91)$ & $(-15.45)$ & $(-16.55)$ & $\ldots$ & -15.40 & -14.97 & -15.07 & -14.59 \\
\hline UGC6817 & 27.11 & -11.82 & -12.08 & -12.68 & -13.50 & -13.71 & -13.84 & -13.76 & -12.89 & -12.31 & -11.92 & -10.67 \\
\hline ESO294-G010 & 26.47 & -8.41 & -8.86 & $\ldots$ & $(-11.14)$ & $\ldots$ & $(-11.99)$ & $\ldots$ & -11.85 & -11.37 & -10.50 & $\ldots$ \\
\hline ESO410-G005 & 26.42 & -7.93 & -9.23 & $\ldots$ & $(-11.53)$ & $\ldots$ & $(-12.25)$ & $\ldots$ & -12.97 & -11.97 & -12.49 & -12.09 \\
\hline ESO540-G032 & 27.67 & -8.14 & -9.11 & $\ldots$ & $(-11.40)$ & $\ldots$ & $(-12.26)$ & $\ldots$ & -11.79 & -10.97 & $\ldots$ & $\ldots$ \\
\hline F08D1 & 27.78 & $\ldots$ & -8.94 & $\ldots$ & $\ldots$ & $\ldots$ & $\ldots$ & $\ldots$ & -13.12 & -12.82 & -13.77 & -13.26 \\
\hline UGC8091 & 26.60 & -11.36 & -11.36 & -11.65 & -12.15 & -12.38 & -12.43 & -12.66 & -11.53 & -11.21 & -10.79 & -10.82 \\
\hline HS117 & 26.61 & -7.74 & $\ldots$ & $(-8.98)$ & $(-10.69)$ & $(-11.49)$ & $(-12.02)$ & $\ldots$ & -11.59 & -10.97 & $\ldots$ & $\ldots$ \\
\hline UGC5666 & 27.93 & -15.38 & -15.51 & $(-16.32)$ & $(-16.77)$ & $(-16.98)$ & $(-17.10)$ & $\ldots$ & -16.68 & -16.31 & -15.94 & -16.01 \\
\hline IKN & 27.84 & $\ldots$ & $\ldots$ & $\ldots$ & $\ldots$ & $\ldots$ & $\ldots$ & $\ldots$ & $\ldots$ & $\ldots$ & $\ldots$ & $\ldots$ \\
\hline M81-DwA & 27.68 & -10.28 & -10.42 & $\ldots$ & $\ldots$ & $\ldots$ & $\ldots$ & $\ldots$ & -11.85 & -11.00 & -7.64 & -10.36 \\
\hline KDG61 & 27.84 & -8.81 & -9.67 & -12.78 & -13.20 & -13.83 & -14.10 & -14.38 & -13.43 & -12.85 & $\ldots$ & $\ldots$ \\
\hline UGC5442 & 27.84 & $\ldots$ & -9.70 & $\ldots$ & $\ldots$ & $\ldots$ & $\ldots$ & $\ldots$ & -13.20 & -12.77 & -10.38 & -12.43 \\
\hline KDG73 & 27.84 & -9.08 & -9.35 & $\ldots$ & $\ldots$ & $\ldots$ & $\ldots$ & $\ldots$ & -12.26 & -10.95 & $\ldots$ & -10.99 \\
\hline KKR03 & 26.41 & -7.94 & -8.42 & -9.14 & -9.94 & -10.23 & -10.18 & -10.61 & $\ldots$ & $\ldots$ & $\ldots$ & $\ldots$ \\
\hline KKH037 & 27.65 & -9.30 & -9.82 & $(-11.21)$ & $(-12.09)$ & $(-12.51)$ & $(-12.81)$ & $\ldots$ & -12.33 & -11.84 & -11.47 & -11.39 \\
\hline ККH086 & 27.03 & -7.71 & -8.76 & -9.96 & -10.74 & -11.26 & -11.33 & -10.91 & $\ldots$ & $\ldots$ & $\ldots$ & $\ldots$ \\
\hline KKH098 & 26.90 & -9.35 & -10.08 & -11.00 & -11.81 & -12.02 & -12.39 & -12.31 & $\ldots$ & 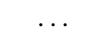 & $\ldots$ & \\
\hline KKR25 & 26.35 & $\ldots$ & $\ldots$ & $\ldots$ & $\ldots$ & $\ldots$ & $\ldots$ & $\ldots$ & $\ldots$ & $\ldots$ & $\ldots$ & $\ldots$ \\
\hline NGC 2366 & 27.53 & -14.87 & -15.03 & $(-15.77)$ & $(-16.06)$ & $(-16.26)$ & $(-16.30)$ & $\ldots$ & -15.71 & -15.34 & -15.52 & -15.71 \\
\hline NGC 3741 & 27.55 & -12.32 & -12.50 & -12.95 & -13.51 & -13.73 & -13.83 & -14.02 & -12.95 & -12.51 & -11.78 & -11.44 \\
\hline NGC 4163 & 27.36 & -11.93 & -12.37 & -13.37 & -14.22 & -14.61 & -14.86 & -14.94 & -14.09 & -13.62 & -13.23 & -12.78 \\
\hline UGC4483 & 27.53 & -11.71 & -11.78 & $(-12.51)$ & $(-12.82)$ & $(-12.82)$ & $(-12.90)$ & $\ldots$ & -11.92 & -11.14 & -9.28 & -11.09 \\
\hline UGC8201 & 28.35 & -13.81 & -14.03 & -14.56 & -15.29 & -15.47 & -15.55 & -15.51 & -14.64 & -14.22 & -13.79 & -13.02 \\
\hline UGC8508 & 27.06 & $\ldots$ & $\ldots$ & -12.63 & -13.32 & -13.63 & -13.79 & -13.94 & -13.07 & -12.55 & -12.45 & -12.42 \\
\hline UGC8833 & 27.47 & -10.85 & -11.05 & -11.80 & -12.55 & -12.86 & -13.02 & -12.64 & -12.33 & -12.17 & -11.47 & -11.22 \\
\hline UGCA292 & 27.79 & -11.44 & -11.49 & -12.13 & -12.20 & -12.38 & -12.60 & -12.61 & -12.53 & -11.59 & -11.48 & -13.03 \\
\hline UGCA438 & 26.74 & -11.23 & -11.37 & $(-11.69)$ & $(-12.46)$ & $(-13.00)$ & $(-13.15)$ & $\ldots$ & -12.65 & -12.17 & -11.96 & -11.32 \\
\hline UGC5428 & 27.72 & $\ldots$ & -9.87 & $(-11.34)$ & $(-12.52)$ & $(-13.02)$ & $(-13.30)$ & $\ldots$ & -12.94 & -12.44 & -11.26 & -12.79 \\
\hline Sex A & 25.60 & -11.75 & -11.87 & $(-12.27)$ & $(-12.53)$ & $(-12.56)$ & $(-12.66)$ & & -11.91 & -11.45 & -10.90 & -9.24 \\
\hline ESO321-G014 & 27.50 & -10.93 & -11.31 & $(-12.20)$ & $(-12.75)$ & $(-13.04)$ & $(-13.21)$ & $\ldots$ & -12.89 & -12.48 & -10.48 & -11.56 \\
\hline ESO540-G030 & 27.61 & -8.20 & -9.31 & $\ldots$ & $(-11.34)$ & $\ldots$ & $(-10.38)$ & $\ldots$ & -12.01 & -11.68 & -10.13 & $\ldots$ \\
\hline IC5152 & 26.58 & -13.35 & -13.69 & $(-14.41)$ & $(-14.95)$ & $(-15.27)$ & $(-15.42)$ & $\ldots$ & $\ldots$ & -14.53 & $\ldots$ & -15.31 \\
\hline Sculptor-dE1 & 28.07 & $\ldots$ & $\ldots$ & $\ldots$ & $\ldots$ & $\ldots$ & $\ldots$ & $\ldots$ & $\ldots$ & $\ldots$ & $\ldots$ & $\ldots$ \\
\hline UGC5373 & 25.67 & -10.76 & -10.99 & -11.72 & -12.74 & -13.03 & -13.14 & -13.25 & -12.31 & -11.93 & $\ldots$ & -11.26 \\
\hline UGC4305 & 27.65 & -15.00 & -15.11 & $(-15.78)$ & $(-16.10)$ & $(-16.18)$ & $(-16.41)$ & $\ldots$ & -15.72 & -15.45 & -15.37 & -15.45 \\
\hline UGC4459 & 27.79 & -12.41 & -12.57 & -13.16 & -13.68 & -13.95 & -14.06 & -14.04 & -13.56 & -13.14 & -12.80 & -13.63 \\
\hline UGC5139 & 27.95 & -13.09 & -13.26 & $(-14.04)$ & $(-14.44)$ & $(-14.66)$ & $(-14.70)$ & $\ldots$ & -14.07 & -13.53 & $\ldots$ & -13.24 \\
\hline UGC5336 & 27.79 & -12.79 & -12.92 & -13.39 & -13.86 & -14.24 & -14.41 & -14.39 & -13.60 & -13.30 & $\ldots$ & -14.65 \\
\hline
\end{tabular}

Notes. All photometry is given as AB absolute magnitudes and corrected for Milky Way extinction.

a Distance modulus.

b Numbers within parentheses are for the Johnson-Cousins filters, numbers without parentheses are for the SDSS system.

\subsection{Predicting Integrated Luminosities from the $\mathrm{SFH}$}

We derive the expected luminosity for each galaxy within the area covered by the HST footprint, by inputting the measured SFHs into a population synthesis code (see Wyder 2001 for an early example of this method). To be consistent with the derivation of the SFH we adopt the Flexible Stellar Population Synthesis (FSPS; Conroy et al. 2009; Conroy \& Gunn 2010) model (ver. 2.3, revision 60) as our fiducial model. This code uses the same Girardi et al. (2000) stellar evolutionary tracks for the main sequence up to the asymptotic giant branch (AGB) phase. The default FSPS models use the AGB star isochrones of Marigo et al. (2008), with modifications as suggested in Conroy \& Gunn (2010). To maintain consistency with the derivation of the SFH from resolved stars, we have used FSPS without these modifications; the effects of changing the AGB isochrones 


\begin{tabular}{lcc}
\multicolumn{3}{c}{ Table 3 } \\
\hline \hline Band & $\lambda_{\text {eff }}$ & $A_{\lambda} / E(B-V)_{\mathrm{MW}}$ \\
& $(\AA)$ & \\
\hline FUV & 1528.1 & 7.9 \\
NUV & 2271.1 & 8.1 \\
$u$ & 3546.0 & 5.0 \\
$U$ & 3571.2 & 5.0 \\
$B$ & 4344.1 & 4.2 \\
$g$ & 4669.6 & 3.8 \\
$V$ & 5455.6 & 3.2 \\
$r$ & 6156.2 & 2.8 \\
$R$ & 6441.6 & 2.6 \\
$i$ & 7471.6 & 2.1 \\
$I$ & 7993.8 & 1.9 \\
$z$ & 8917.4 & 1.5 \\
$3.6 \mu \mathrm{m}$ & 35416.6 & 0.0 \\
$4.5 \mu \mathrm{m}$ & 44826.2 & 0.0 \\
$5.6 \mu \mathrm{m}$ & 56457.2 & 0.0 \\
$8 \mu \mathrm{m}$ & 78264.8 & 0.0 \\
\hline
\end{tabular}

on the predicted SED will be discussed in Section 4.1. PostAGB evolution utilizes the tracks of Vassiliadis \& Wood (1994). For main sequence and giant stars, the stellar spectra in FSPS models are from BaSeL 3.1 (Westera et al. 2002). Spectra of TP-AGB stars are from the empirical library of Lançon \& Mouhcine (2002), with extensions redward of $2.5 \mu \mathrm{m}$ using Aringer et al. (2009) for carbon-rich TP-AGB stars and the PHOENIX "BT-SETTL" spectral library for oxygen-rich TPAGB stars. The spectra of post-AGB stars are from Rauch (2002) and the spectra of OB and Wolf-Rayet stars are from Smith et al. (2002). We use a Salpeter IMF from 0.1 to $100 M_{\odot}$ for consistency with the CMD fitting. We consider a fiducial metallicity of $0.2 Z_{\odot}$ for all models. This value is consistent with the measured gas-phase metallicities, the metallicities expected from the mass-metallicity relation, and estimates of the metallicity from the CMD fitting. The metallicity has only a modest effect on the UV and optical luminosities, as we discuss further in Section 5.1, and we do not expect significant effects from limiting the models to a single metallicity. These models do not include dust attenuation or emission, except for the circumstellar envelopes of AGB stars. The stellar synthesis models implicitly assume that the CMD is fully sampled, and thus neglect stochastic effects due to small numbers of stars at high stellar masses or rare evolutionary phases.

We have also considered the population synthesis models of Bruzual (2007), which are based on the "Padova 1994" (Bertelli et al. 1994) stellar evolutionary tracks, supplemented by the Marigo et al. (2008) evolutionary tracks for TP-AGB stars. The main difference between Girardi et al. (2000) and "Padova 1994" is a warmer (and bluer) giant branch in the former (Bruzual \& Charlot 2003). There are some differences between the FSPS models and Bruzual (2007) in the stellar spectral libraries used. However, we find that using the Bruzual (2007) models instead of our fiducial models does not significantly affect the predicted SED (except in the Spitzer 5.8 and $8 \mu \mathrm{m}$ bands, see Section 4), and does not change our conclusions.

In detail, we generate SPS models having constant SFR (of $1 M_{\odot} \mathrm{yr}^{-1}$ ) from zero age to the duration of each of the time bins used in the SFH reconstruction, and zero SFR thereafter. The resulting spectra of the SPS models (with each model corresponding to a single time bin in the $\mathrm{SFH}$ ) are then interpolated logarithmically in time to a set of ages $t-t_{i}$

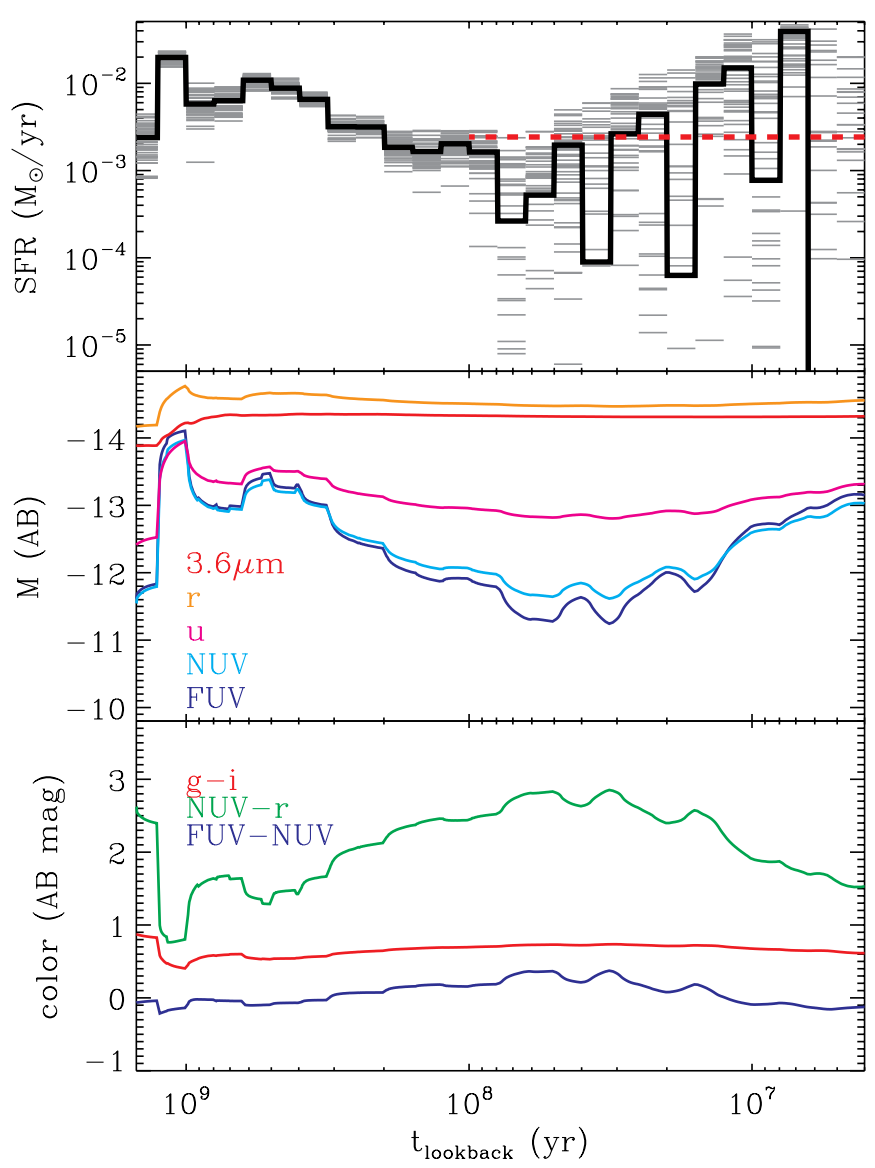

Figure 2. Top: an example star formation history. The black histogram shows the best-fit SFH determined from fits to the optical stellar CMD for NGC 4163, at higher temporal resolution than shown in Weisz et al. (2011). The gray histograms show the MC realizations of the SFH. The red dashed line shows the SFR averaged over the past $100 \mathrm{Myr}\left(\langle\mathrm{SFR}\rangle_{8}\right)$. Middle: an example of the modeled luminosity evolution. The colored lines give the absolute $\mathrm{AB}$ restframe magnitude in several bands as a function of lookback time, expected given the SFH in the top panel. Bottom: modeled color evolution for the same galaxy.

(A color version of this figure is available in the online journal.)

where $t_{i}$ is the beginning time of the bin, and the appropriate normalization is applied:

$$
\ell_{\lambda}^{\bmod }(t)=\sum_{i}^{N_{\text {bin }}} \operatorname{SFR}_{i} \hat{\ell}_{i, \lambda}\left(t-t_{i}\right) e^{-\tau_{\lambda}\left(t-t_{i}\right)},
$$

where $\mathrm{SFR}_{i}$ is the SFR in time bin $i$ from the CMD based $\mathrm{SFH}, \hat{\ell}_{i, \lambda}\left(t^{\prime}\right)$ is the luminosity at wavelength $\lambda$ of the truncated constant SFR SPS model at age $t^{\prime}$, and $\tau_{\lambda}\left(t^{\prime}\right)$ is the dust extinction optical depth at wavelength $\lambda$ towards stars of age $t^{\prime}$. In order to match the reddening model used when deriving the SFH from the CMD (Section 2.4) the maximum of $\tau_{\lambda}\left(t-t_{i}\right)$ is taken to linearly decrease from $\tau_{V}=0.5$ for $t-t_{i} \leqslant 40 \mathrm{Myr}$ to $\tau_{V}=0$ at $t-t_{i}=100 \mathrm{Myr}$. To account for the distribution of extinctions toward stars of a given age in the Sextans A differential extinction model (Section 2.4 and Dolphin 2002), the spectrum of each bin, $\hat{\ell}_{i, \lambda}\left(t^{\prime}\right)$, is divided into a large number of equal pieces, each piece is extinguished with a different $\tau_{V}$ uniformly distributed up to the maximum for that time bin, and the pieces are then summed. We note that this makes little difference from simply assuming one average value for $\tau_{V}$ per bin, regardless of the number of pieces. As the sample is primarily composed of low-mass, low-metallicity dwarf galaxies, the shape of the 
extinction curve is taken to be that of the Small Magellanic Cloud (SMC; Pei 1992), and we neglect scattering. The resulting $\ell_{\lambda}^{\bmod }(t)$ are convolved with the appropriate filter transmission curves to determine the broadband luminosity $L_{\lambda}^{\bmod }(t)$, which we refer to as the modeled (with dust) luminosity. We also determine the modeled (intrinsic, dust-free) luminosity in all bands, denoted $L_{0, \lambda}^{\bmod }(t)$ hereafter, by setting $\tau_{\lambda}=0$ for all ages. Stellar masses, accounting for stellar evolutionary effects, are derived in a similar way by replacing $\hat{\ell}_{i, \lambda}\left(t^{\prime}\right)$ with $\hat{m}_{i}\left(t^{\prime}\right)$, the stellar mass of all surviving stars and stellar remnants at age $t^{\prime}$. Typically this stellar mass is $\lesssim 0.15$ dex smaller than the total stellar mass formed over the lifetime of the galaxy.

The SFR and duration of the most recent time bin is altered so that the total mass of stars formed in that bin is distributed over the interval 0 to $4 \mathrm{Myr}$. This is because the derivation of the SFR from the CMD results in stars with ages $<4$ Myr being "assigned" (or fit by stars with) the minimum isochrone age (4 Myr $<t_{\text {lookback }}<5 \mathrm{Myr}$ ). We include an estimate the effects of nebular emission on the broadband luminosities, which we find to be less than 0.05 dex at all wavelengths.

Figure 2 shows an example of the derived luminosity evolution of one galaxy. It is clear that the discrete binning of the SFH has strong effects on the flux evolution, especially at large lookback time when the temporal resolution is longer than the lifetime of the dominant stars. However, the fluctuations in SFR at large lookback times do not significantly affect the present day luminosities. At small lookback times the temporal resolution is better, and the effect of the binning on even the UV luminosity evolution is small. The effects of binning in the SFH are discussed further in Section 2.6.

\subsection{Uncertainties in the SFH}

There are several sources of uncertainty in the measured SFHs that can affect the predicted luminosities. The first source is random uncertainties due to the number of stars in each region of the CMD. Statistical uncertainties on the SFHs are computed through 50 Monte Carlo (MC) realizations. For each MC test, a Poisson random noise generator is used to randomly resample the best fitting model CMD. As a result, the uncertainties on the SFHs account for fluctuations in the number of stars used to derive the SFH in a given time bin. For each galaxy we repeat the analysis described above for different $\mathrm{MC}$ realizations and then compute the dispersion in the resulting flux at each wavelength to obtain an estimate of the uncertainty of the predicted flux at the present day. This procedure also takes into account the covariance between adjacent bins. The typical uncertainty in the predicted FUV flux is $\sim 0.05$ dex, and is unbiased with respect to the FUV luminosity of the best-fitting SFH. This uncertainty decreases to $\sim 0.01$ dex redward of the $u$ band. A similar procedure is used to estimate the uncertainties in $\langle\mathrm{SFR}\rangle_{8}$ and $M_{*}$ as derived from the SFH. Again, because of significant covariance between time bins the uncertainties on time averaged properties, including broadband luminosities, are significantly smaller than implied by quadrature sums of the uncertainties on the SFR in individual bins.

The second source of uncertainties in the SFHs are systematic effects due to differences between stellar models (see, e.g., Dolphin 2012; Charlot et al. 1996; Conroy et al. 2009; Weisz et al. 2011). Estimating the effect of these systematic uncertainties on the predicted fluxes requires deriving the SFH with different stellar models and then predicting the broadband flux with the same model. The differences in the predicted fluxes when using these different stellar models then give an estimate of the systematic uncertainty of the predicted flux. While Weisz et al. (2011) have provided an estimate of the uncertainty in the SFH induced by uncertainties in stellar isochrones, a fair estimate of the resulting uncertainty in the integrated broadband luminosity requires predicting the integrated SED with the same (randomly) modified isochrones. This is beyond the scope of the current paper, but deserves treatment in future analyses. Readers are cautioned that the uncertainties in the physical parameters listed in Table 1 do not include the (often dominant) systematic uncertainties due to changes in the stellar models (Weisz et al. 2011; Dolphin 2012).

The third source of uncertainty in the predicted fluxes is the discrete time binning of the SFHs. At one extreme, the SFR may fluctuate more smoothly than the derived SFH, simply due to the binning in time of a smoothly rising or falling SFH. At the other limit, the true SFR may vary on timescales shorter than the width of a given bin (see, e.g., Eskew \& Zaritsky 2011 for a discussion of the impact of SFRs that vary strongly within a given bin). In general, these limitations will be a significant effect only for the wavelengths that are sensitive to timescales shorter than the resolution of the SFH. For example, the ionizing flux $(\lambda<912 \AA)$ is nearly always sensitive to the SFR on timescales shorter than the temporal resolution of the SFH, and therefore significant artifacts of the binning scheme would be visible in the $\mathrm{H} \alpha$ flux evolution, even at small lookback times. For the broadband wavelengths, however, the temporal resolution is sufficient for the prediction of present day luminosities.

\section{COMPARISON TO OBSERVED SED: AVERAGE DIFFERENCES}

In this section, we compare the modeled luminosities to the observed luminosities. This comparison allows us to (1) verify the reliability of the SFHs, (2) explore the effects of additional galaxy properties on the SED, free from the potentially degenerate effects of SFH, and (3) test the predictions of population synthesis models in the UV and NIR.

In Figure 3 we show the modeled present-day SED for a subset of the galaxy sample, as well as the observed broadband luminosities. We also show the contribution of stars formed in several different broad age bins to the present day SED, and the residuals between the modeled luminosities and the observed luminosities. In general there is good agreement.

In Figure 4 we plot the average differences between the observed luminosities and the modeled luminosities as a function of wavelength, with error bars showing the standard error of the mean. The first thing to note is the excellent agreement in the UV through optical (FUV through $i$ ), where the overall normalization appears to be correct to within $0.1 \mathrm{dex}$, and often much better. The stars used for the SFH determination typically contribute $\sim 40 \%$ of the total luminosity in the $g$ through $V$ bands (though this fraction varies significantly) suggesting that the SFH is correctly inferring the behavior of the remaining $60 \%$ of the flux.

However, in the NIR portions of the spectrum, we see significant systematic differences. Real galaxies appear to be fainter than the models in the Spitzer IRAC 3.6, 4.5 and $5.8 \mu \mathrm{m}$ bands by an average of $\sim 0.2$ dex. As discussed in Conroy et al. (2009) and Mancone \& Gonzalez (2012), the emission in this region of the SED is often dominated by more poorly understood phases of stellar evolution (particularly the TPAGB phase), and it is here that different population synthesis models disagree most strongly. This uncertainty is in contrast 

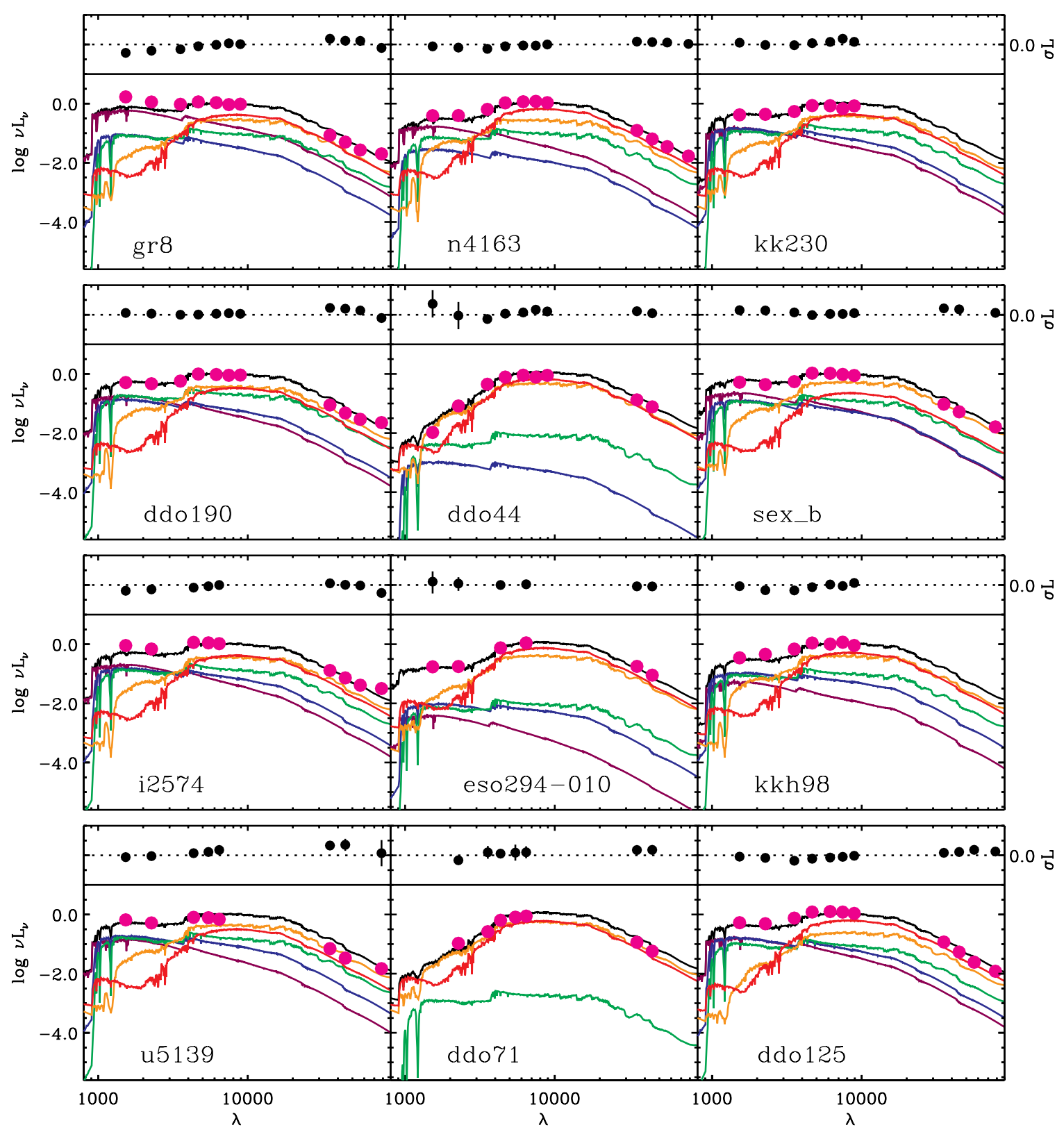

Figure 3. The modeled and observed SED for 12 of the sample galaxies. In each panel, the magenta points show the observed SED for one galaxy, while the black line shows the modeled $t_{\text {lookback }}=0$ SED. The colored lines give the contribution to the present day spectrum of stars formed in different time bins: $6.7<\log t_{l} \leqslant 7.3$ (purple), $7.3<\log t_{l} \leqslant 8.0$ (blue), $8.0<\log t_{l} \leqslant 8.7$ (green), $8.7<\log t_{l} \leqslant 9.4$ (orange), $9.4<\log t_{l} \leqslant 10.15$ (red). All SEDs are normalized by the $5500 \AA$ luminosity of the modeled $t_{\text {lookback }}=0$ SED. At the top of each panel the black points show the residuals between the modeled and observed broadband luminosity $\left(\delta L=\log L_{\mathrm{mod}} / L_{\mathrm{obs}}\right)$, with error bars giving the quadrature sum of the photometric error and the dispersion of the modeled flux from the different MC realizations. The residuals are on the same scale as the SEDs.

(A color version of this figure is available in the online journal.)

to the optical, where the stellar spectra and the evolutionary tracks of the dominant populations are more certain and do not vary as widely between authors. The origin of these systematic offsets will be discussed further in Section 4. Here we note that splitting the sample by stellar mass, which correlates with many other parameters (e.g., metallicity, SFR) yields similar results for galaxies both more and less massive than $10^{7.5} M_{\odot}$

In Table 4 we present, for each wavelength, the average and rms dispersion of the ratio of the modeled luminosity to the observed luminosity, $\log \left(L_{\mathrm{mod}} / L_{\mathrm{obs}}\right)$. We will refer to these ratios in each band as "offsets" or "excesses." In the optical the scatter is low $(\sim 0.1 \mathrm{dex})$, and in all bands is less than 0.2 dex.

\section{DIFFERENCES IN THE NIR}

In this section, we explore the differences between modeled and observed luminosity in the NIR that were found in Section 3. The light in the NIR IRAC bands comes from a number of sources. At 3.6 and $4.5 \mu \mathrm{m}$, the IRAC flux is dominated by the long-wavelength tail of cool luminous AGB, RGB, and RHeB stars, with a modest contribution from interstellar lines and dust. In the longer wavelength 5.6 and $8 \mu \mathrm{m}$ IRAC filters, the flux is increasingly dominated by emission from dust, especially polycyclic aromatic hydrocarbon (PAH) emission.

The average differences between the modeled and observed luminosities in the stellar-dominated $3.6 \mu \mathrm{m}$ and $4.5 \mu \mathrm{m}$ IRAC 

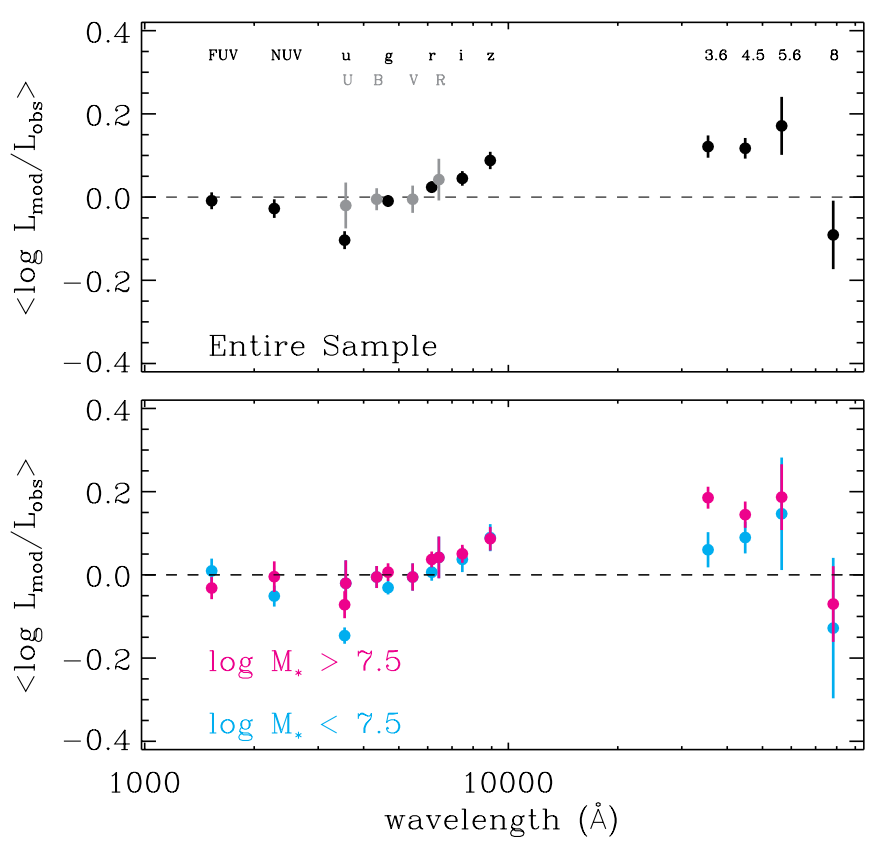

Figure 4. Comparison of observed luminosities to modeled luminosities. Top: average differences between the absolute magnitudes measured from broadband imaging (and corrected for MW extinction) and the absolute magnitudes determined from the CMD-derived SFH and the FSPS models, as function of wavelength for the entire sample. The error bars give the standard error of the mean. The Johnsons-Cousins filters are shown in gray. Bottom: the same, but with the sample split by stellar mass, showing that the offsets are not a strong function of stellar mass or properties that correlate with stellar mass.

(A color version of this figure is available in the online journal.)

Table 4

Average SED Differences

\begin{tabular}{lccc}
\hline \hline Filter & $\left\langle\log L_{\text {mod }} / L_{\text {obs }}\right\rangle$ & $\sigma\left(\log L_{\text {mod }} / L_{\text {obs }}\right)$ & $N_{\text {obs }}$ \\
\hline FUV & -0.009 & 0.125 & 38 \\
NUV & -0.028 & 0.145 & 42 \\
$u$ & -0.104 & 0.099 & 21 \\
$U$ & -0.020 & 0.214 & 15 \\
$B$ & -0.005 & 0.115 & 19 \\
$g$ & -0.009 & 0.066 & 21 \\
$V$ & -0.005 & 0.127 & 15 \\
$r$ & 0.024 & 0.064 & 21 \\
$R$ & 0.042 & 0.213 & 18 \\
$i$ & 0.045 & 0.080 & 21 \\
$z$ & 0.088 & 0.096 & 21 \\
$3.6 \mu \mathrm{m}$ & 0.121 & 0.171 & 41 \\
$4.5 \mu \mathrm{m}$ & 0.117 & 0.161 & 42 \\
$5.6 \mu \mathrm{m}$ & 0.171 & 0.334 & 23 \\
$8 \mu \mathrm{m}$ & -0.091 & 0.411 & 25 \\
\hline
\end{tabular}

bands are important to consider in light of the widespread use of NIR photometry for stellar mass determinations (e.g., Lee et al. 2006; Berg et al. 2012; Meidt et al. 2012), and ongoing uncertainty in the treatment of TP-AGB stars in population synthesis models. In the 3.6, 4.5, and $5.6 \mu \mathrm{m}$ bands, and to a lesser extent in the $i$ band, there is a significant offset, in the sense that the observed NIR luminosities are fainter than predicted by our fiducial model. There are several possible reasons for these offsets, which may also contribute to the scatter in the ratio of modeled to observed luminosity. First, systematic uncertainties in the ancient SFH (that are not included in the MC-derived uncertainties, Section 2) may cause offsets in the NIR bands. The ancient SFH is most strongly constrained by the fainter, redder

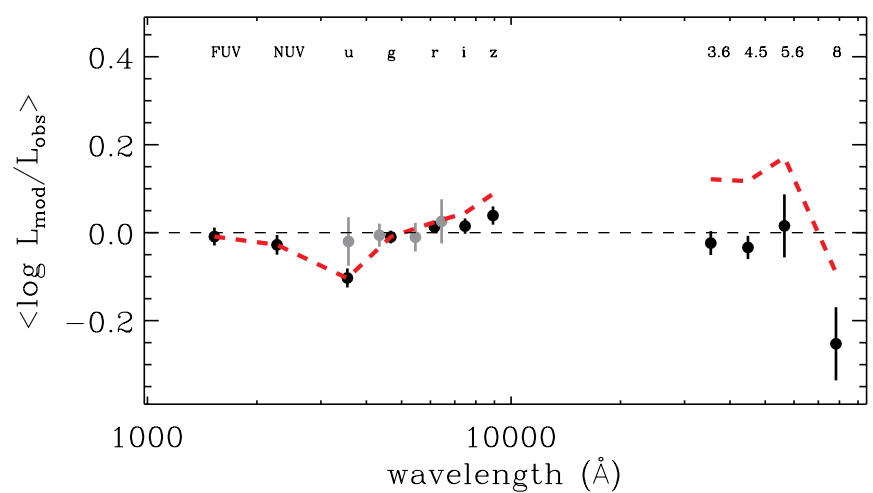

Figure 5. Comparison of observed luminosities to modeled luminosities with reduced contribution to the NIR from TP-AGB stars, as suggested by Conroy \& Gunn (2010). Symbols are as in Figure 4. The red dashed lines shows the results of Figure 4, where the TP-AGB contribution is not reduced. The systematic offsets in the NIR have been substantially reduced.

(A color version of this figure is available in the online journal.)

RGB stars, but we find no trend of the NIR offsets with the depth of the HST CMD; a thorough exploration of this possibility will require deeper CMDs (as are available for local group galaxies or the Magellanic clouds). The second, more likely, explanation is related to the treatment of uncertain phases of stellar evolution in population synthesis models, especially the TP-AGB phase. This is discussed in more detail below.

\subsection{The Impact of AGB Star Prescriptions}

The contribution of TP-AGB stars to the NIR spectrum of galaxies is the subject of much debate (Maraston 2005; Bruzual 2007; Conroy et al. 2009; Kriek et al. 2010; Girardi et al. 2010; Zibetti et al. 2013). Both the evolutionary paths and the NIR spectra of these stars are poorly constrained, and thus we are not surprised to find significant disagreements between the predicted and observed NIR luminosity. To explore possible origins of the observed offsets, we have considered population synthesis models with different treatments of the TP-AGB phase. While this breaks the consistency between the population synthesis modeling and the derivation of the SFH, tests by Girardi et al. (2010) and Melbourne et al. (2012) have found that the CMD-based SFH of many of our sample galaxies are relatively insensitive to the treatment of the AGB phase, and produce nearly identical SFHs even when AGB stars are excluded from the fit. This is because there are few of them relative to the RGB, and so they carry less weight in the SFH determination.

We first examine the effect of modifying the TP-AGB isochrones from the Marigo et al. (2008) treatment, as suggested by Conroy \& Gunn (2010). This modification was made to better match the optical/NIR colors of Magellanic cloud globular clusters at the ages dominated by TP-AGB stars. The net effect of this change is to significantly reduce the contribution of TP-AGB stars to the NIR SED. Using the FSPS models with modified AGB isochrones to predict the SED as in Section 2.5, we compare the newly predicted luminosities to the observed luminosities in Figure 5. This figure can be directly compared to Figure 4. Even though these galaxies are not post-starbursts, nor young enough that the NIR SED is dominated by TP-AGB stars (see, e.g., Kriek et al. 2010; Zibetti et al. 2013), there is a significant effect on the predicted IRAC luminosities such that the (average) agreement with the observed luminosities is improved. The median ratio of the predicted to observed 
luminosities at 3.6 and $4.5 \mu \mathrm{m}$ has decreased from 0.22 and 0.15 dex to 0.02 and $-0.03 \mathrm{dex}$, respectively. This change in predicted luminosities also demonstrates that modeled NIR mass-to-light ratios are sensitive to these different treatments of the evolution of TP-AGB stars at least at the 0.2 dex level.

In the Spitzer NIR bands, we found that the modeled SEDs consistently overpredict the $3.6,4.5$, and $5.8 \mu \mathrm{m}$ luminosities by $\sim 0.2$ dex on average. However, if the evolution of TP-AGB stars is altered from that of Marigo et al. (2008) then it is possible to obtain much better agreement. This conclusion is qualitatively consistent with Melbourne et al. (2012), who found that the Marigo et al. (2008) models tend to significantly overpredict (by a factor of two or more) the number and total luminosity of luminous TP-AGB stars that are present in the HST $H$-band imaging of many of these same galaxies. When using the Girardi et al. (2010) evolutionary tracks for TP-AGB stars, Melbourne et al. (2012) found that the numbers of TP-AGB stars were better matched to observations, though the total TP-AGB luminosity was still overpredicted by factors of approximately two. Fortunately, the fraction of the total $H$-band light due to TP-AGB stars is relatively small for these galaxies, and because this overprediction of TP-AGB luminosity is largely offset by an underproduction of RHeB luminosity in the models relative to the data, Melbourne et al. (2012) found the effect of this systematic overprediction of TP-AGB luminosity on the total $H$-band luminosity to be small. However, at 3.6 and $4.5 \mu \mathrm{m}$, where the fraction of the total luminosity due to TP-AGB stars is likely to be larger than at $1.6 \mu \mathrm{m}$ (e.g., Bruzual 2007), and the fraction due to $\mathrm{RHeBs}$ smaller than at $1.6 \mu \mathrm{m}$, an overestimate of the TP-AGB luminosity may have larger consequences for the total luminosity than at $H$ band. A detailed comparison of the predictions of different evolutionary tracks and spectral libraries will be the subject of a future work.

With a decrease in the contribution of TP-AGB stars to the integrated NIR SED, the observed $8 \mu \mathrm{m}$ luminosities are larger than the modeled $8 \mu \mathrm{m}$ luminosities, consistent with a small contribution of unmodeled dust in this band. However, when the galaxies are split by stellar mass (or alternatively metallicity) as in the bottom panel of Figure 4, the lowmass, low-metallicity galaxies still show an apparent deficit of modeled $8 \mu \mathrm{m}$ luminosity. Since these galaxies are expected to have a smaller abundance of PAH grains and lower PAH emission (e.g., Madden 2000; Hogg et al. 2005; Marble et al. 2010; Wu et al. 2011), this deficit of the modeled $8 \mu \mathrm{m}$ luminosities may not necessarily be due to the lack of modeled dust emission from the interstellar medium (ISM), but instead to the uncertain spectra of AGB stars at these wavelengths (perhaps related to the un-modeled circumstellar dust around these stars, e.g., Srinivasan et al. 2011).

\section{IMPACT OF METALLICITY, STOCHASTICITY, AND DUST ATTENUATION}

\subsection{Metallicity}

In Section 2.5 we fixed the stellar metallicity at $0.2 Z_{\odot}$, due to uncertainties in the stellar metallicities of the sample galaxies. In this section, we examine the effect of variations of the true metallicity from this assumed value on the modeled luminosities. We do this using a constant SFR as input to FSPS, and determine the SED for a variety of assumed metallicities. The ratio of the resulting luminosity to the luminosity of the $Z=0.2 Z_{\odot}$ model as a function of $Z$ over the plausible range

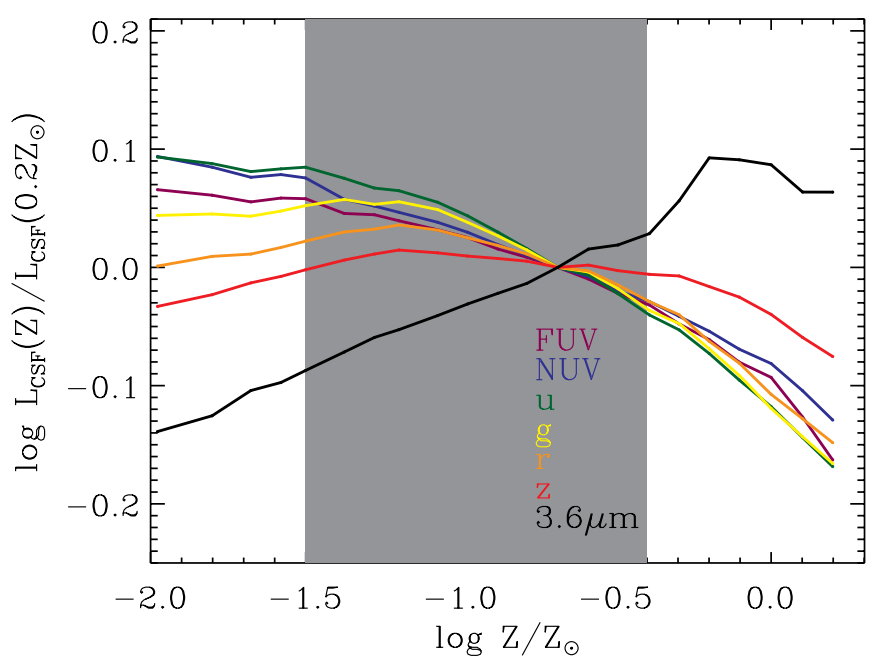

Figure 6. The luminosity in several broadbands of a constant SFR model as a function of stellar metallicity, relative to the $Z=0.2 Z_{\odot}$ models assumed in Section 2.5. The gray shaded region indicates the plausible range of current gas-phase metallicity of our sample galaxies, inferred from emission lines and the NIR luminosity-metallicity relation.

(A color version of this figure is available in the online journal.)

of metallicities for our low mass sample of galaxies is shown in Figure 6.

For the lower metallicity galaxies in our sample, our assumed metallicity may lead to underestimates of the true luminosity by up to $\sim 0.1$ dex in the UV. Conversely, the IRAC luminosities of low metallicity galaxies may be overestimated by as much as much as 0.1 dex, or more if the stellar metallicities of the stars contributing most strongly in these bands are significantly lower than the gas-phase metallicity. However, there are few galaxies at the very lowest metallicities, and tests assuming $Z=0.1 Z_{\odot}$ for the stellar metallicity indicate that the average offsets change by less than $0.05 \mathrm{dex}$ (and that $\left\langle L_{\mathrm{mod}} / L_{\mathrm{obs}}\right\rangle$ increases slightly in the IRAC NIR bands). Combined with the agreement seen in Figure 4, this suggests that the effect of stellar metallicity on the comparisons is minimal, though metallicity variations may contribute a small amount $(\lesssim 0.05$ dex $)$ to the scatter of individual offsets at a given wavelength.

\subsection{Stochastic Sampling of the IMF}

At very low SFR, or alternatively for a small mass of "young" stars, it is possible that stochastic sampling of the IMF and the cluster mass function can lead to an apparent deficit of massive stars, due to their relative rarity (Cerviño \& Valls-Gabaud 2003; Cerviño \& Luridiana 2009). This effect has been shown to be important in the interpretation of the $\mathrm{H} \alpha$ flux of dwarf galaxies, which arises ultimately from the hydrogen ionizing emission of $\gtrsim 20 M_{\odot} \mathrm{O}$ and early B stars (Lee et al. 2009; Fumagalli et al. 2011; Eldridge 2012). This stochastic sampling of the IMF may also affect the UV flux of galaxies with low SFR. However, since the UV emission arises from more numerous, lower mass stars, the effect will always be less important than for $\mathrm{H} \alpha$.

Because we have used population synthesis models that assume a fully sampled IMF when constructing the predicted SEDs and UV fluxes, our models do not include the scatter and bias in flux that is caused by stochasticity. On the other hand, the uncertainties on the SFH derived from the CMDs do take this stochasticity into account implicitly in the fitting process, and generally rely on more numerous stars of lower masses. Thus, if stochastic effects are important for the UV luminosity, 


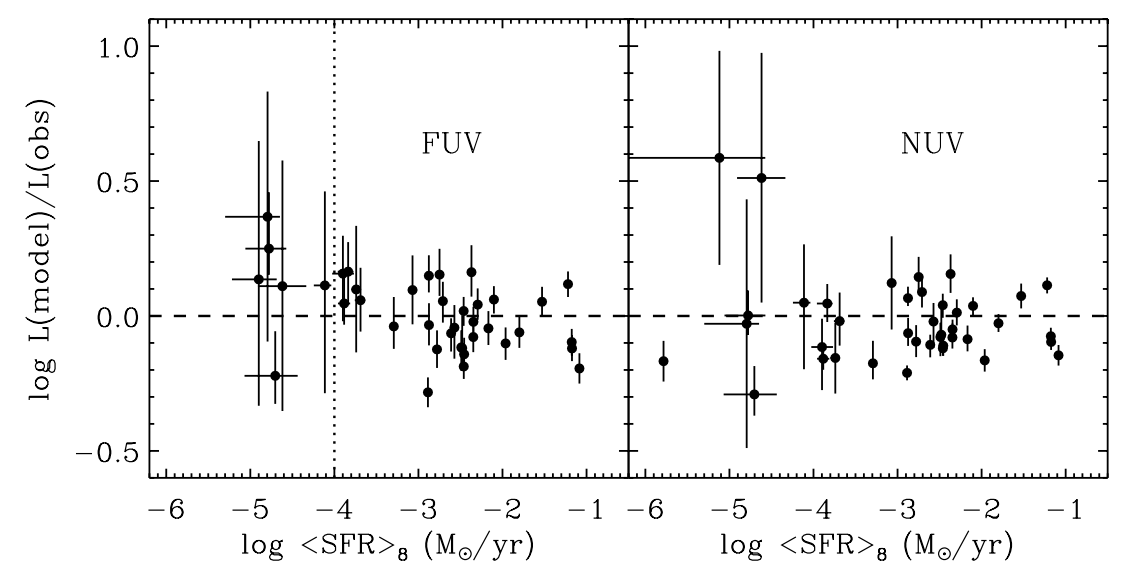

Figure 7. $\langle\mathrm{SFR}\rangle_{8}$, the star formation rate averaged over $100 \mathrm{Myr}$ inferred from HST CMDs, is plotted against the difference between the observed and modeled fluxes at FUV (left) and NUV (right). The dotted line in the left-hand panel marks the SFR below which stochastic effects might be expected to become important. Horizontal dashed lines mark perfect agreement between modeled and observed luminosities.

we would expect a difference between the observed and predicted SED that is larger than the uncertainty in the predicted UV flux inferred from the MC realizations (in the sense that the observed SED is fainter than predicted on average). We would also expect an increased uncertainty or scatter in the predicted UV flux if stochastic effects were to be included. These differences would anti-correlate with the SFR of the galaxy, or more properly with the mass of stars formed over some characteristic UV emitting lifetime, because at higher SFRs there should be more complete sampling of the upper IMF.

The contribution of stochasticity to the luminosity differences is explored indirectly in Figure 7, where we plot the FUV and NUV luminosity differences versus $\langle\mathrm{SFR}\rangle_{8}$. While the scatter in luminosity differences appears to increase slightly at lower $\langle\mathrm{SFR}\rangle_{8}$, we find no clear correlation, suggesting that stochastic effects do not play a large role in the observed UV luminosities of these galaxies. While a proper accounting of the effect of stochasticity on the average and scatter in UV luminosity would require modeling the drawing of individual stars from the IMF and cluster mass function given a total mass of stars formed in the various time intervals (da Silva et al. 2012 though note that the SFHs used in our study implicitly include sampling of the cluster mass function), the comparisons shown in Figure 7 are sufficient to rule out the stochasticity as a major contributor to individual offsets between the observed and modeled UV luminosities.

\subsection{Dust Attenuation}

In Section 2.5 we modeled the luminosities both including the effects of the differential reddening model that is required to match the observed optical CMD and without this differential reddening (i.e., the intrinsic, dust-free luminosity). The reddened luminosities were found to provide a good match to the observed luminosities in the UV through optical bands. In this section, we compare the extinctions inferred from the reddened and unreddened model luminosities to commonly-used independent estimates of the dust attenuation based on the observed ratio of the IR to UV luminosity.

The effective total extinction at any wavelength can be derived from the model luminosities of Section 2.5 by

$$
A_{\text {model }, \lambda}=-2.5 \log \left(\frac{L_{0, \lambda}^{\mathrm{mod}}}{L_{\lambda}^{\mathrm{mod}}}\right)
$$

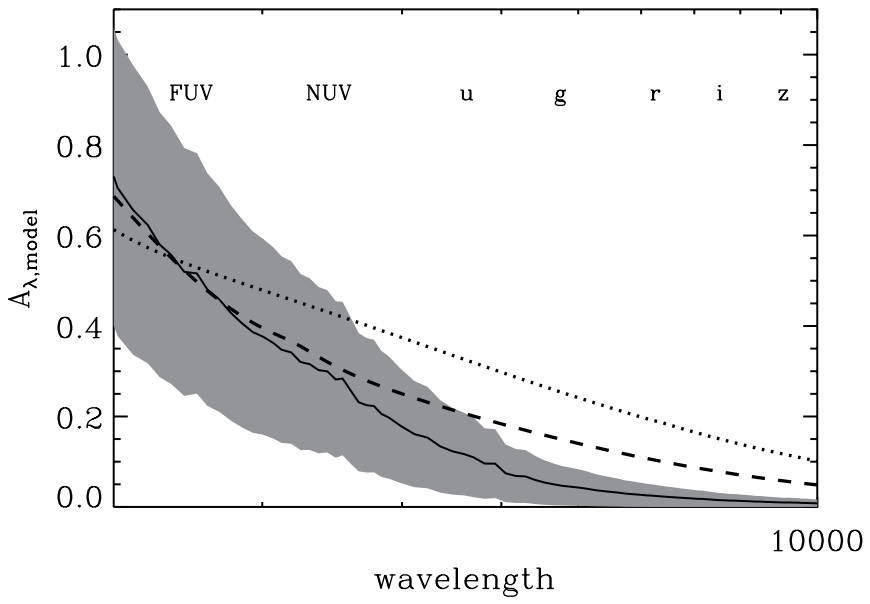

Figure 8. The average model effective extinction as a function of wavelength for the sample (solid line). The gray shaded region shows the rms scatter in the extinction among the galaxies (due to variations of the SFH in combination with the differential extinction model). For comparison, a Calzetti et al. (2000) attenuation curve and the Pei (1992) SMC extinction curve are shown as the dotted and dashed lines respectively. Both curves have been normalized by the average $A_{\text {fuv,mod. }}$.

where $L_{0, \lambda}^{\text {mod }}$ is the intrinsic model luminosity derived from the SFH without extinction by dust and $L_{\lambda}^{\bmod }$ is the luminosity derived including both $\mathrm{SFH}$ and the differential extinction model. The average and scatter of these modeled attenuations for the sample are shown as a function of wavelength in Figure 8. Note that, because the reddening only affects stars younger than $100 \mathrm{Myr}$, and because we have assumed the relatively steep attenuation curve of the SMC, the extinction of bands redward of NUV are small. Also, because the differential extinction model is fixed, the scatter is due entirely to the different SFHs among the sample galaxies.

In Figure 9 we compare $A_{\text {model }}$ for the FUV, NUV, and $u$ bands to the FUV attenuation $A_{\text {fuv }}$ inferred from the ratio of $24 \mu \mathrm{m}$ to FUV luminosity based on the calibration of Hao et al. (2011; Section 2.3) In nearly every case $A_{\text {fuv }}$ is significantly smaller than the attenuation derived from the CMD based SFH and differential extinction model. However, the calibration of $A_{\text {fuv }}$ given by Hao et al. (2011) is largely based on much more massive and dusty galaxies, where older stars may contribute a larger fraction of the dust luminosity, and where the effective 

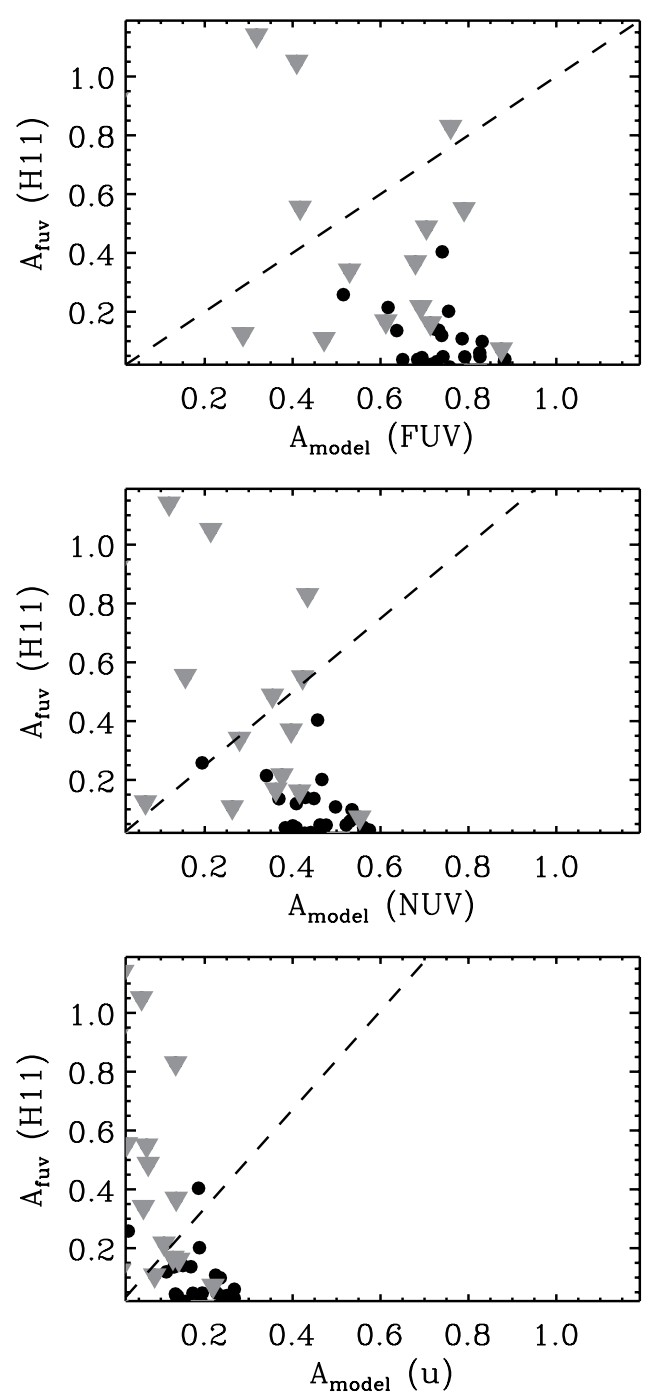

Figure 9. Comparison of the model effective extinction in the FUV, NUV, and $u$ bands to $A_{\text {fuv }}$ estimated from the ratio of $24 \mu \mathrm{m}$ to FUV luminosity following Hao et al. (2011). Gray triangles indicate upper limits to $A_{\text {fuv }}$ based on $24 \mu \mathrm{m}$ upper limits. Dashed lines are the expected relation for a Calzetti et al. (2000) attenuation curve.

attenuation curve is significantly shallower or flatter than we have derived in Figure 8.

A more direct comparison of the modeled extinctions to the infrared luminosity can be made by integrating the difference between the spectra modeled with and without differential extinction (the $\ell_{\lambda}^{\text {mod }}$ of Section 2.5) and assuming that this extinguished luminosity is reradiated in the infrared. In Figure 10 we compare this extinguished luminosity to the IR luminosity derived from Spitzer MIPS observations by Dale et al. (2009), both with and without corrections for the smaller aperture of the HST data. This comparison is only possible for the 20 galaxies in the sample with detections in all MIPS bands.

We find that the total extinguished model luminosity is larger than the observed IR luminosity by a factor of four on average. While this may be a result of modeled extinctions that are larger than the true extinction, tests have shown that assuming zero extinction results in very poor fits to the optical CMD. Alternatively, the assumption that all extinguished light is reradiated in the IR is likely to be incorrect. Scattering by dust will serve to decrease the amount of extinguished light in our model SEDs that is ultimately absorbed by dust (e.g.,

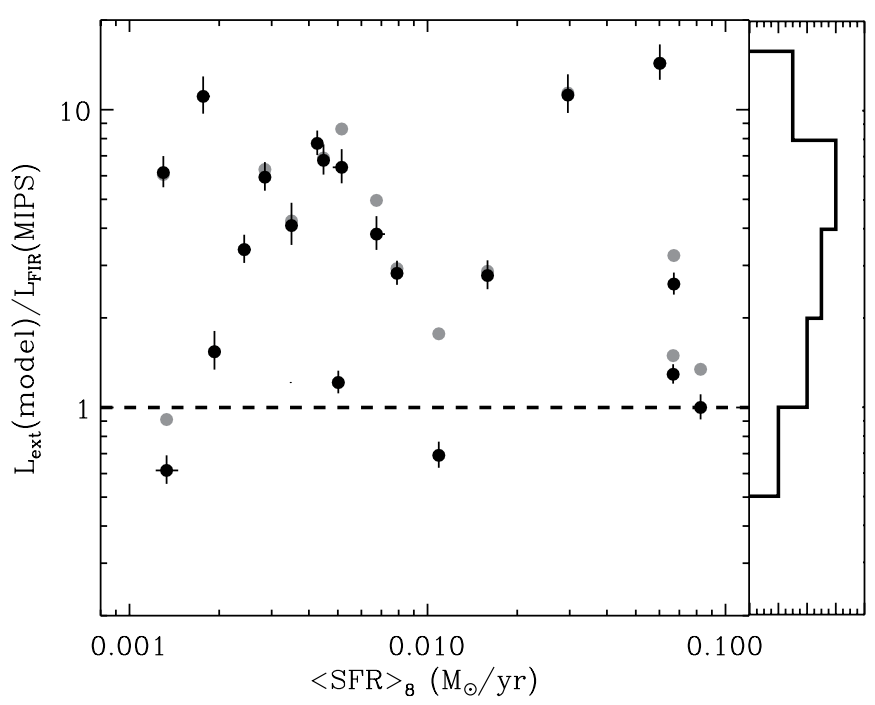

Figure 10. Comparison of the observed total FIR luminosity, calculated from MIPS luminosities following Dale et al. (2009), to the total extinguished luminosity predicted given the extinction model. Vertical error bars only include photometric uncertainties. Gray points include a correction for the fraction of young star light falling outside the HST aperture. The histogram in the right panel indicates the number of galaxies in logarithmic bins of the ratio of the extinguished model luminosity to the observed IR luminosity.

Witt \& Gordon 2000). However, because the scattered light is primarily at UV wavelengths, a significant contribution of scattered light would then result in a poor match between the observed and modeled UV luminosities in Figure 4. Assuming the attenuation curves of Witt \& Gordon (2000) for a clumpy, cloudy geometry with an SMC extinction curve and a $V$ band extinction of $0.25 \mathrm{mag}$ (as in the differential extinction model of Section 2.5) yields absorbed IR luminosities that are consistent with the observed IR luminosities on average (though with large scatter), but also results in modeled FUV luminosities that are brighter than the observed luminosity by $\sim 0.2$ dex on average.

It is possible that some moderate amount of differential extinction, combined with the relatively gray attenuation curves of Witt \& Gordon (2000), might adequately match the optical CMD without predicting too much IR luminosity and that corresponding changes in the recent SFH would maintain the agreement we find between the observed and modeled UV and optical SEDs. A detailed exploration of this possibility would benefit greatly from multi-band stellar photometry and CMDs to further constrain the differential extinction, as are being obtained in M31 as part of the Panchromatic Hubble Andromeda Treasury survey (Dalcanton et al. 2012).

\subsubsection{IRX- $\beta$}

The intrinsic UV color of galaxies has implications for measurement of dust attenuation via restframe UV colors using the so-called IRX- $\beta$ diagram (Meurer et al. 1999), which relates the IR to UV luminosity ratio (IRX, a measure of attenuation) to the observed UV spectral slope $\beta$ (where $\left.f_{\lambda} \propto \lambda^{-\beta}\right)$. Over the last decade, there has been increasing evidence that normal galaxies are shifted to redder UV colors in this diagram than starburst galaxies (e.g., Seibert et al. 2005; Kong et al. 2004; Johnson et al. 2007b; Gil de Paz et al. 2007; Dale et al. 2007, 2009; Boquien et al. 2012), with significant implications for the measurement of attenuation in large samples of high-redshift galaxies (e.g., Reddy et al. 2012; Smit et al. 2012). It is often proposed that variations in the SFH are the 


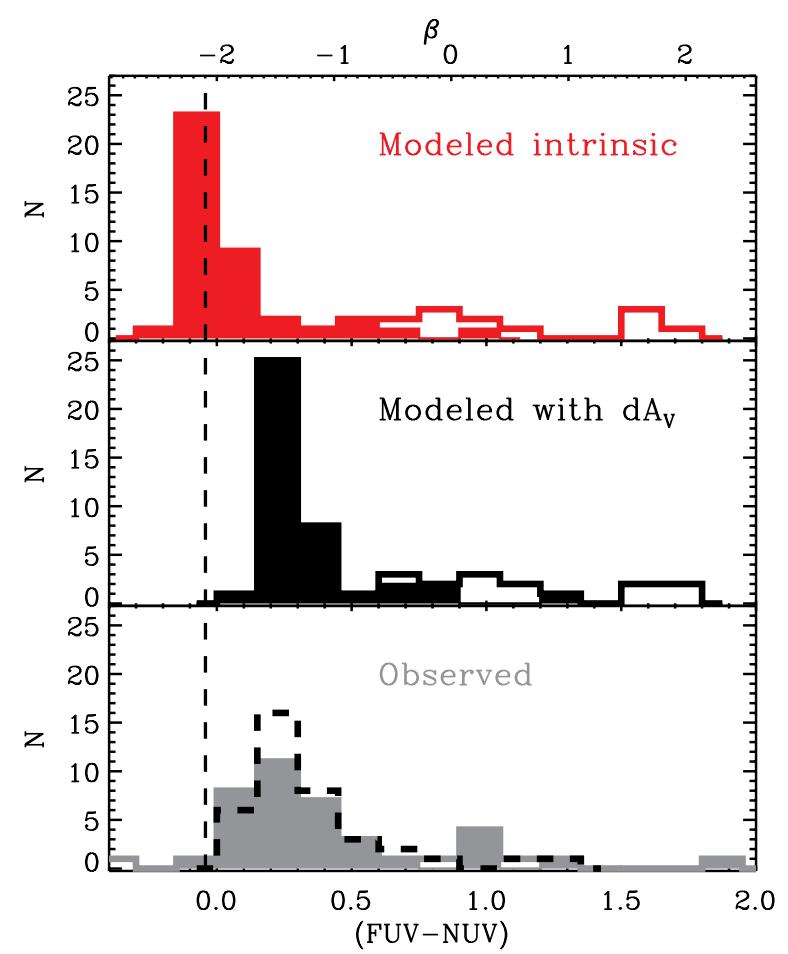

Figure 11. The distribution of FUV - NUV color for the sample galaxies. Top: the intrinsic FUV - NUV color derived from the luminosities modeled without differential attenuation. The filled histogram includes all sample galaxies not classified as dwarf spheroidals. Middle: as for the top, but for the modeled FUV - NUV color including differential extinction. Bottom: the observed FUV - NUV color distribution for all galaxies with detections in both bands is shown in gray. The filled histogram is for only the galaxies that are not dwarf spheroidals. The dashed black histogram shows the distribution of modeled FUV - NUV colors including differential extinction and a contribution from photometric and model uncertainties in the FUV - NUV color. In all panels the vertical dashed line marks the intrinsic, dust-free color for a constant SFR model. The top axis indicates the UV spectral slope $\beta$, estimated as $\beta=2.3$ (FUV $-\mathrm{NUV})-2$.

(A color version of this figure is available in the online journal.)

cause for this scatter, since more passive galaxies may have a redder intrinsic UV color.

Using the SFHs derived in Section 2.5, we explore this possibility. This sample is particularly well suited to such a study as many of the sample galaxies have low IRX but have been found to be shifted to redder UV colors than starburst galaxies (Dale et al. 2009). First, we determine the intrinsic FUV - NUV color implied by the SFHs of Section 2.4; that is, the color that is obtained without application of the differential reddening model. The distribution of these intrinsic UV colors are shown as the red histogram in Figure 11, where the filled histogram does not include the galaxies classified as dwarf spheroidals. We find a small scatter of these intrinsic colors around the value predicted for constant SFR.

We have also determined the distribution of UV colors implied by the SFHs and the differential reddening model, shown as the black histogram in Figure 11, finding that differential reddening shifts the galaxies to redder UV colors (by 0.3 mag or 0.6 in $\beta$ ) but without significantly increasing the scatter in color. Finally, we construct the distribution of observed UV colors (the gray histogram in Figure 11), which is centered close to the model distribution including differential extinction, but has a somewhat higher dispersion. This higher observed dispersion may be caused by uncertainties in both the modeled colors and the observed colors. To investigate this possibility, we add
UV color shifts to the modeled FUV - NUV color which are drawn, for each galaxy, from a normal distribution with standard deviation given by the quadrature sum of the model and photometric uncertainties in UV color. Including these uncertainties, a two-sided Kolmogorov-Smirnoff test is unable to reject the hypothesis that the modeled and observed UV colors are drawn from the same distribution. Some additional small uncertainty in the modeled UV colors may arise from the differential reddening model assumed in the CMD analysis and our assumed extinction curve shape.

The blue peak of the UV color distribution in the top panel of Figure 11 indicates that the SFH alone is not sufficient to explain the red offset in the UV colors of galaxies with low IRX, unless the recent SFHs are systematically incorrect. However, the middle and bottom panels of Figure 11 indicate that the inclusion of differential extinction is sufficient to explain the observed UV color distribution well.

\section{THE RELATIONSHIP BETWEEN UV LUMINOSITY AND SFR}

The UV luminosity of galaxies is commonly used as a tracer of the SFR both in the local universe and at high redshifts, where it is redshifted into the optical. The conversion between UV luminosity and SFR is typically made assuming that the SFR is approximately constant for at least 100 Myr (Madau et al. 1998; Kennicutt 1998). However, if the SFR varies on shorter timescales, then the conversion between luminosity and SFR becomes more complicated, and the conversion depends on the exact distribution of stellar ages. This complication has long been known (Kennicutt 1998), but galaxies that undergo significant variations in their SFR on these timescales are thought to be rare. There are two classes of galaxies where the assumption of a constant recent SFR is known to fail. The first are starburst (and post-starburst) galaxies, which are thought to have enhanced recent SF due to interactions (e.g., Hopkins et al. 2006; Wild et al. 2009). The second are dwarf galaxies, for which rapid variation may be driven by stochasticity in the cluster formation process at low star formation rates (e.g., Fumagalli et al. 2011), or by the effects of feedback in low mass halos (e.g., Stinson et al. 2007). Sub-regions of galaxies also demonstrate variations in SFR on short timescales and episodic star formation (e.g., Gogarten et al. 2009; Huang et al. 2013).

The model luminosities determined in Section 2.5 allow us to explore the effect of realistic, observationally constrained SFHs on the ratio of UV luminosity to the SFR averaged over a given timescale. We define the SFR conversion factor $\epsilon_{\lambda, T}$ via the equation

$$
\langle\mathrm{SFR}\rangle_{T}=\left(\lambda \mathrm{L}_{\lambda}\right) / \epsilon_{\lambda, T},
$$

where $T$ is the log of the averaging timescale in years. The FUV luminosity is typically assumed to trace the SFR over $100 \mathrm{Myr}$, approximately the lifetime of FUV emitting stars. We will thus consider $\epsilon_{\mathrm{FUV}, 8}$. The modeled FUV luminosity accounts for the effects of SFH, but not dust attenuation, metallicity, or other effects as discussed in Section 3. Because both the SFR and the modeled UV luminosity are derived from the same SFH, the results we obtain in this section do not involve the observed luminosity. Furthermore, our conclusions do not require that the derived SFHs (Section 2.4) are exactly correct in detail for each galaxy, only that they are plausible and not systematically biased.

In Figure 12, we present the distribution of $\epsilon_{\mathrm{FUV}, 8}^{\mathrm{mod}}$, i.e., the ratio of the modeled FUV luminosity to $\langle\mathrm{SFR}\rangle_{8}$. We 


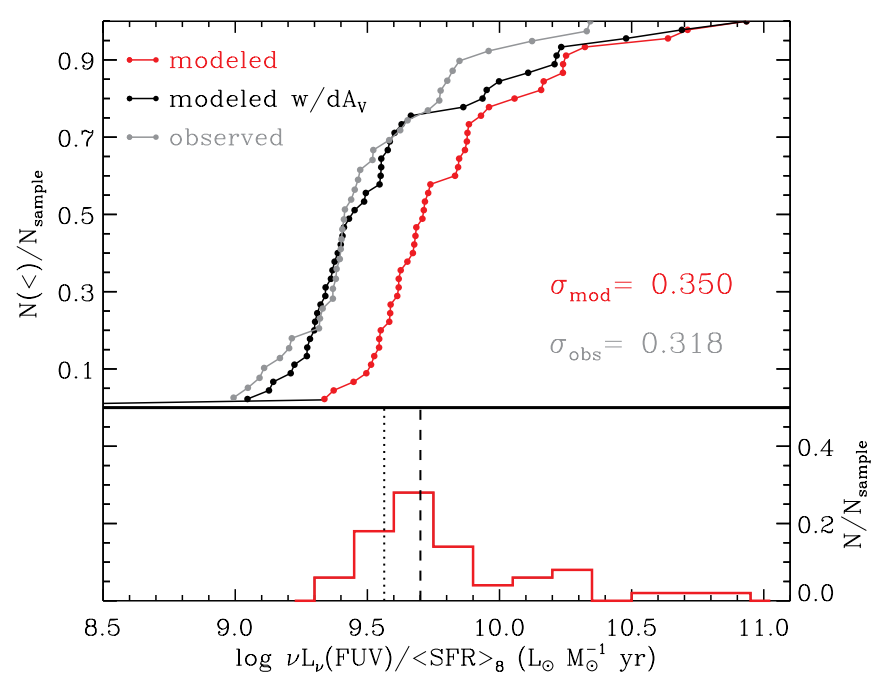

Figure 12. The modeled FUV to SFR conversion factors for the sample. The distribution of $\epsilon_{\mathrm{FUV}, 8}^{\mathrm{mod}}$, the ratio of the modeled intrinsic FUV luminosity to $\langle\mathrm{SFR}\rangle_{8}$, is shown as a red histogram in the bottom panel, while the connected red points in the top panel show the cumulative distribution. The gray connected points show the cumulative distribution of $\epsilon_{\mathrm{FUV}, 8}^{\mathrm{obs}}$, the ratio of the observed $\mathrm{FUV}$ luminosity to $\langle\mathrm{SFR}\rangle_{8}$, while the black connected points show the cumulative distribution of the ratio of the modeled luminosity including dust to $\langle\mathrm{SFR}\rangle_{8}$. The vertical dotted line marks the conversion factor of Kennicutt (1998), while the dashed line is the constant SFR conversion factor for the population synthesis models adopted here with $0.2 Z_{\odot}$ metallicity. The expected conversion factors for exponentially declining, $0.2 Z_{\odot}$ metallicity models with $e$-folding times of 1-13 Gyr and ages more than 1 Gyr are all to the right of the dashed line.

(A color version of this figure is available in the online journal.)

show this ratio for both the modeled intrinsic luminosities and for the modeled luminosities including differential reddening. The $\epsilon_{\mathrm{FUV}, 8}^{\mathrm{mod}}$ that we derive can be compared directly to the conversion factor of Kennicutt (1998), plotted as the dashed line in Figure 12. Kennicutt (1998) derived a single conversion factor assuming solar metallicity, a nearly constant SFR, and the same IMF as we have adopted (see also Madau et al. 1998). Figure 12 shows that the observationally constrained SFH, on its own, induces significant scatter in $\epsilon_{\mathrm{FUV}, 8}$. Depending on the SFH of a particular galaxy, the conversion between UV luminosity and $\langle\mathrm{SFR}\rangle_{8}$ can vary by an order of magnitude. For our sample of galaxies, the rms dispersion in $\epsilon_{\mathrm{FUV}, 8}^{\mathrm{mod}}$ is a factor of $\sim 2$.

The scatter in the ratio of $\langle\mathrm{SFR}\rangle_{8}$ to the modeled FUV luminosity is 0.3 dex, while the scatter in the ratio of the observed luminosity to the modeled FUV luminosity (including SFH effects) is only 0.14 dex. This suggests that the SFH dominates the scatter in the conversion from FUV luminosity to $\langle\mathrm{SFR}\rangle_{8}$ in this sample. Additional effects such as photometric uncertainties and metallicity variations, and the distribution of dust attenuation, can only contribute an additional 0.14 dex of scatter.

The conversion factor of Kennicutt (1998) shown in Figure 12 is lower than the median $\epsilon_{\mathrm{FUV}, 8}$ that we derive. This can be traced to the different assumptions made in Kennicutt (1998) about the metallicity and the duration of star formation, and is not due to the details of the recent SFH. To better match the properties of our sample, we derive an alternative version of the (Kennicutt 1998) conversion factor as follows. We use the population synthesis models described in Section 2.5, assuming $Z=0.2 Z_{\odot}$ and constant SFR lasting $>10 \mathrm{Gyr}$, and derive a value of the conversion factor that is larger than the Kennicutt (1998) value, and that is approximately the median of $\epsilon_{\mathrm{FUV}, 8}^{\mathrm{mod}}$. This larger value is due to the combined effects of lower metallicity and a longer period of constant SFR than adopted by Kennicutt (1998).

In models with exponentially declining SFRs, the conversion factor $\epsilon_{\mathrm{FUV}, 8}$ varies with the specific SFR of the model. In such $\tau$-models, $\epsilon_{\mathrm{FUV}, 8}$ increases as the specific SFR decreases, and is almost always larger than in the case of constant SFR. ${ }^{11}$ Thus, if the SFHs were well described by smoothly evolving $\tau$-models, we would expect to find a correlation between the conversion factor and some tracer of the specific SFR, such as broadband color. In Figure 13 we show the color (and specific SFR) dependence of $\epsilon_{\mathrm{FUV}, 8}^{\mathrm{mod}}$. The much smoother relationships between $\epsilon_{\mathrm{FUV}, 8}$ and broadband colors that are expected for exponentially declining models are plotted as dashed lines in Figure 13 . We find only a very weak correlation between $\epsilon_{\mathrm{FUV}, 8}^{\mathrm{mod}}$ and broadband color. That is, for the SFHs considered here, it is difficult to predict $\epsilon_{\mathrm{FUV}, 8}^{\mathrm{mod}}$ from broadband color information alone, even when the effects of dust attenuation and metallicity variations are not included. The lack of correlation is due to the fact that $\epsilon_{\mathrm{FUV}, 8}$ traces a shorter timescale of SFH than the broadband colors, and that for our sample the SFH on short timescales is not well correlated with the SFH on longer timescales.

\subsection{The Timescale of UV Emission}

It is often assumed that the FUV and NUV luminosity trace the SFR over timescales of $\sim 100$ and $\sim 200 \mathrm{Myr}$, respectively. However, this assumption is only valid for a constant SFR. We have demonstrated that the SFH of galaxies can cause significant variation in $\epsilon_{\mathrm{FUV}, 8}$, the ratio of the UV luminosity to the SFR averaged over $100 \mathrm{Myr}$. This is due to galaxy by galaxy variations in the distribution of the ages of stars contributing to the FUV luminosity. A single representative timescale for the UV luminosity is therefore difficult to define for a sample of galaxies with diverse SFH. It is important to understand this difficulty (and correct for it if possible) when comparing SFR determinations between samples of galaxies with different SFH.

With strong constraints on the SFH of galaxies from optical CMDs, it is possible to explore the age distribution of stars contributing to the UV luminosity of real galaxies, and to define a characteristic timescale for each galaxy. In Figure 14 we show, for each galaxy, the cumulative fraction of the current UV luminosity as a function of the age of the contributing stars (i.e., the fraction of the current UV luminosity that is produced by stars that are older than a given lookback time). For a constant SFR this cumulative fraction shows an almost linear rise in the FUV, as nearly equal amounts of the current FUV luminosity are contributed by stars in equal logarithmic age bins, up to $\sim 100 \mathrm{Myr}$ ages (see also Kennicutt \& Evans 2012). However, few of the sample galaxies display this behavior. At the extremes, galaxies that reach a cumulative fraction of 1 at large lookback times have nearly all of their UV luminosity coming from relatively old stars. Conversely, galaxies that reach a cumulative fraction of 1 at smaller lookback times have a larger contribution to the current UV luminosity from young stars.

In Figure 14 we see that the fraction of the total FUV luminosity that is produced by stars younger than $10 \mathrm{Myr}$ ranges from $0 \%$ to $\sim 60 \%$. The fraction of the total FUV luminosity that is produced by stars older than $100 \mathrm{Myr}$ ranges from less than $5 \%$ to $100 \%$. Similarly, the fraction of the total NUV luminosity produced by stars older than 200 Myr ranges from less than 5\% to $100 \%$.

\footnotetext{
11 Conversion factors smaller than the constant SFR expectation are only possible in $\tau$-models when the age is very young, $\lesssim 300$ Myr.
} 

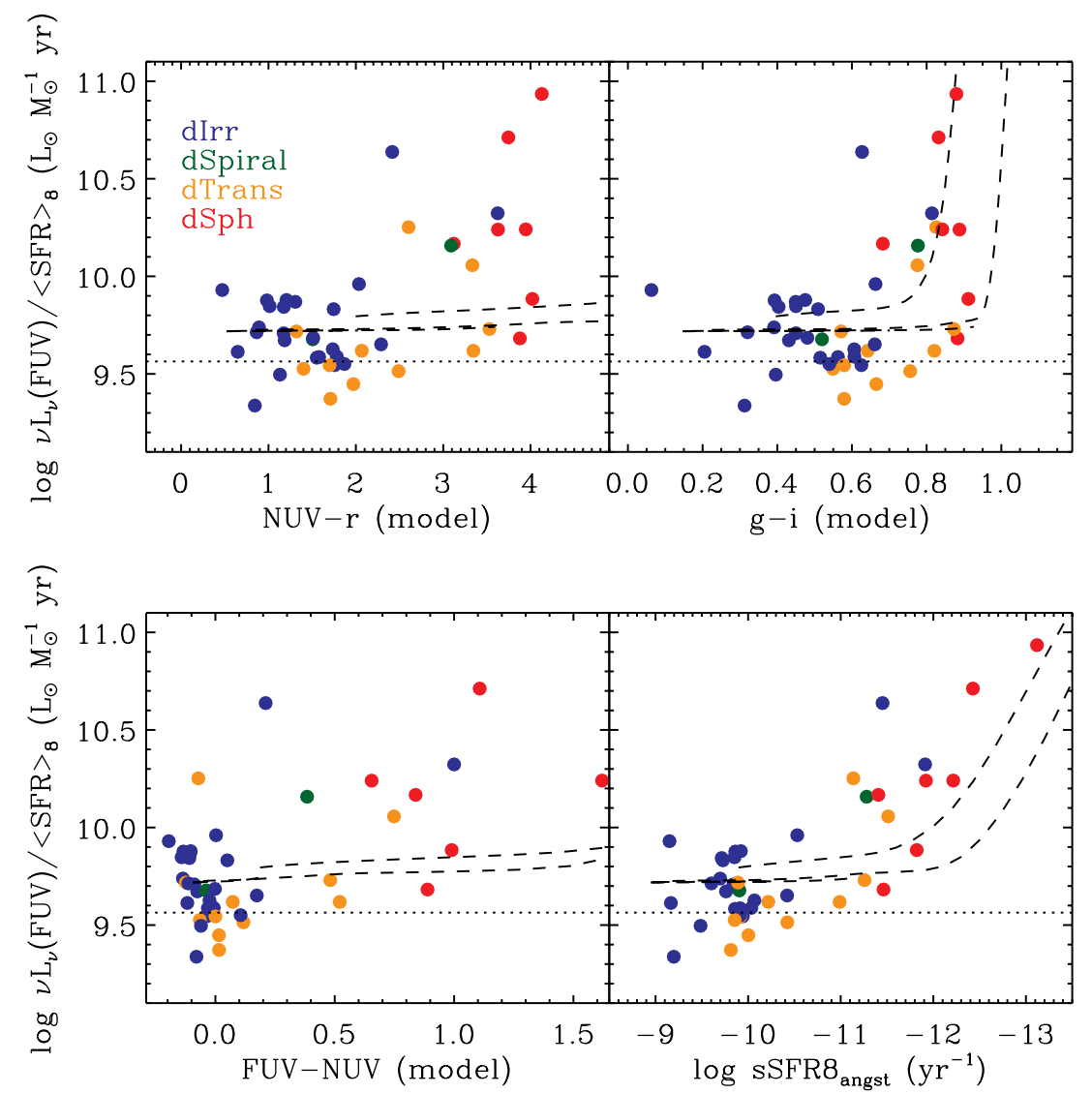

Figure 13. Color dependence of the modeled FUV to SFR conversion factor. Top left: the ratio of the modeled FUV luminosity to $\langle\mathrm{SFR}\rangle_{8}$ (i.e., $\epsilon_{\mathrm{FUV}}^{\mathrm{mod}}{ }_{8}$ ), as a function of the modeled NUV $-r$ color. Different morphological types are shown in different colors: dSph (red), dTrans (orange), dSpiral (green), and dI (blue). Top right: the same, but plotted against the modeled $g-i$ color. Bottom left: the same, but for the FUV - NUV color. Bottom right: the same, but plotted against the specific SFR derived from the SFH. The dotted line in each panel marks the conversion factor of Kennicutt (1998), while the dashed lines are the expectations for population synthesis models with several different rates of exponential decline.

(A color version of this figure is available in the online journal.)

The age distribution of UV emitting stars depends on the SFH, and so it may be possible to identify coarse measures of SFH that correlate with the cumulative fractions given in Figure 14. We focus on the NUV $-r$ color, an observable that is often used as a proxy for the coarse, long-term SFH of a galaxy (Salim et al. 2005, 2007; Schiminovich et al. 2007). We have separated the galaxies in Figure 14 by the predicted NUV $-r$ color. This separation shows that for galaxies with NUV $-r>2.86$, more than $50 \%$ of the modeled FUV luminosity arises from stars older than 100 Myr. For galaxies with NUV $-r<1.1$, more than 50\% of the modeled FUV luminosity is contributed by stars younger than $\sim 16$ Myr.

We have also separated the galaxies by morphological type. Morphological type is correlated with NUV $-r$ color, and so we see a similar difference between galaxies of the dSph type (for which a majority of the FUV flux arises from stars older than 100Myr) and dIrr. The behavior in the NUV is similar, but shifted to larger timescales.

We can use these cumulative luminosity fractions to construct, for each galaxy, a measure of the typical age of the UV producing population. This measure, $\tau_{50}$, is defined as the age where $50 \%$ of the luminosity in a given band comes from younger stars, and $50 \%$ from older stars. In Figure 14, $\tau_{50}$ is the lookback time where a colored line crosses the thin dotted line marking $50 \%$ of the total UV luminosity. Figure 14 shows that $\tau_{50}$ ranges from $\sim 7 \mathrm{Myr}$ to $1 \mathrm{Gyr}$ for the FUV and from $\sim 7 \mathrm{Myr}$ to $3 \mathrm{Gyr}$ for the
Table 5

Modeled $\tau_{\mathrm{UV}}$ as Function of NUV $-r$ Color

\begin{tabular}{lccccc}
\hline \hline Band & Flux Fraction & $a$ & $b$ & $\sigma\left(\log \tau_{\text {UV }}\right)$ & $R$ \\
\hline 6FUV & $50 \%$ & 6.567 & 0.506 & 0.417 & 0.700 \\
6FUV & $80 \%$ & 7.029 & 0.558 & 0.344 & 0.853 \\
NUV & $50 \%$ & 6.453 & 0.687 & 0.342 & 0.831 \\
NUV & $80 \%$ & 7.215 & 0.652 & 0.184 & 0.962 \\
a & & & & & \\
\hline
\end{tabular}

Note. ${ }^{a}$ Fits are of the form $\log \tau_{\mathrm{Band}, \text { Fraction }}=a+b(\mathrm{NUV}-r)$.

NUV. The median $\tau_{50}$ for the FUV and NUV are 26 and $38 \mathrm{Myr}$, respectively.

In the top left panel of Figure 15 we show $\tau_{50}$ for the FUV band against the modeled NUV $-r$ color. There is a good correlation; redder galaxies typically have larger $\tau_{50 \text {,FUV }}$ in the FUV, though several galaxies show much lower $\tau_{50 \text {,FUv }}$ for their color than would be expected from the general trend. The top right panel of Figure 15 shows $\tau_{50}$ for the NUV band. Here the correlation is even stronger, though of course all the $\tau_{50 \text {, NUv }}$ are shifted to larger lookback times than $\tau_{50, \text { NUv }}$. In Table 5 we present the results of linear fits to the relationships between NUV $-r$ color and both $\tau_{50}$ and $\tau_{80}$ for the GALEX bands.

The observed relationship between NUV $-r$ and $\tau_{50}$ is different than expected for exponentially declining models, which are 


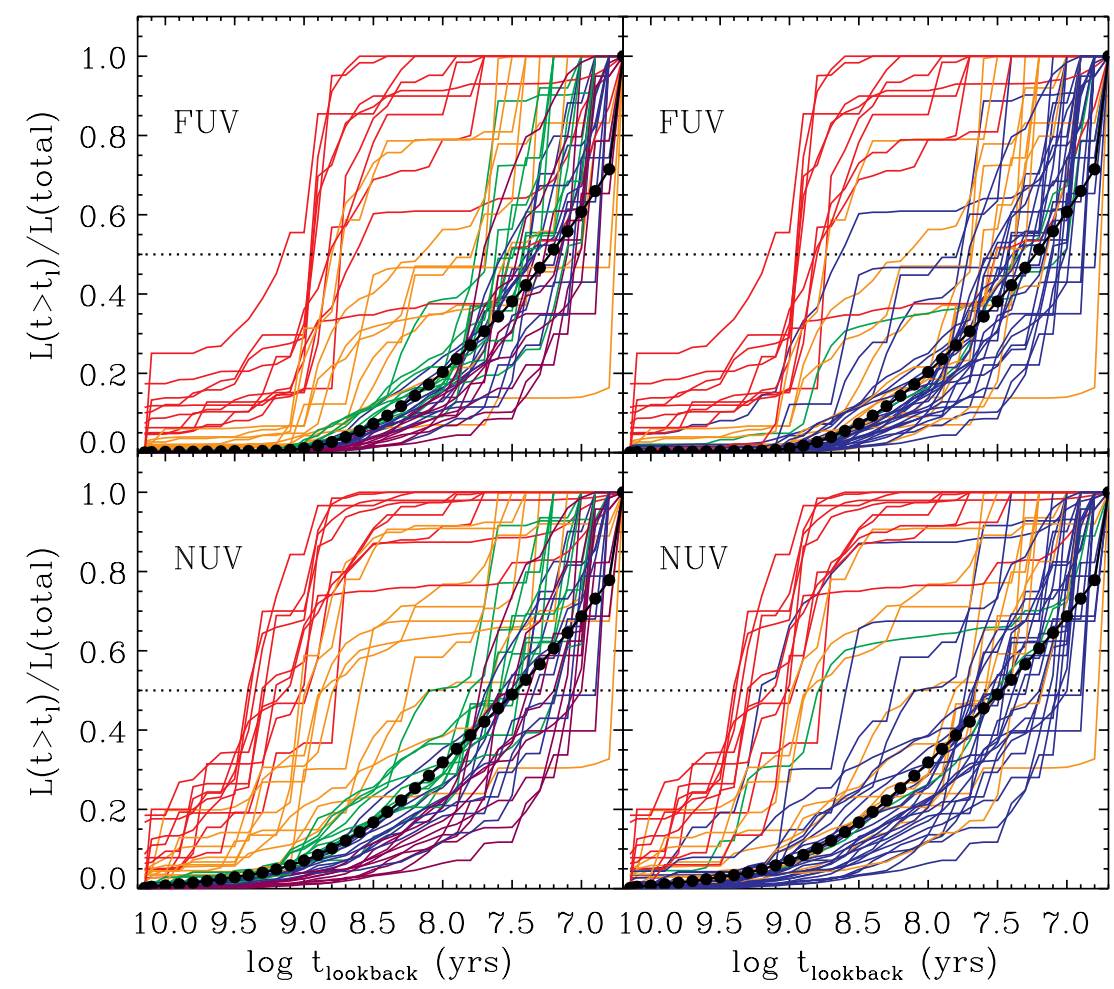

Figure 14. The source of UV luminosity in galaxies. The colored lines give the cumulative fraction of current FUV (top) or NUV (bottom) luminosity produced by stars older than $t_{\text {lookback}}$. The black solid points are the expectation for constant SFR. The dotted line denotes $50 \%$ of the current UV luminosity. Left: the color coding is by the modeled NUV $-r$ color, with bins in color of $0.05<\mathrm{NUV}-r<1.1$ (purple), $1.1<\mathrm{NUV}-r<1.48$ (blue), $1.48<\mathrm{NUV}-r<1.86$ (green), $1.86<\mathrm{NUV}-r<2.86$ (orange), $2.86<\mathrm{NUV}-r<4.0$ (red). Right: the color coding is by morphological type, showing dIrr (blue), dSpiral (green), dTrans (orange) and dSph (red).

(A color version of this figure is available in the online journal.)

also shown in Figure 15. The observed relation is more linear than for exponentially declining models, and the minimum $\tau_{50}$ is lower for some galaxies than the minimum of the exponentially declining models. In the FUV, approximately 12 of the sample galaxies ( $25 \%$ of the sample) have $\tau_{50, \mathrm{FUV}}<16 \mathrm{Myr}$, which is the value expected for constant SFR. Furthermore, for 10 of the sample galaxies $(20 \%) \tau_{50, \text { FUV }}$ is greater than $100 \mathrm{Myr}$, the canonical FUV timescale, and these galaxies have bluer NUV $-r$ colors than exponentially declining models with the same $\tau_{50, \mathrm{FUV}}$.

\subsection{Summary}

In this section, we have demonstrated that SFH causes significant scatter in $\epsilon_{\mathrm{FUV}, 8}$, the ratio of the FUV luminosity to $\langle\mathrm{SFR}\rangle_{8}$. Comparison with the observed FUV luminosity indicates that variations of the SFH dominate the total scatter in $\epsilon_{\mathrm{FUV}, 8}$ for this sample of galaxies. The scatter in $\epsilon_{\mathrm{FUV}, 8}$ does not correlate strongly with UV/optical colors or specific SFR.

We also calculated the age distribution of stars that contribute to the FUV and NUV luminosity. We find a broad range of characteristic ages $\tau_{50}$, where $\tau_{50}$ is defined such that stars younger than $\tau_{50}$ contribute $50 \%$ of the total UV luminosity. For the FUV band, $\tau_{50}$ ranges from less than $16 \mathrm{Myr}$ (for a quarter of our sample) to greater than $100 \mathrm{Myr}$ (for one fifth of our sample). This is a sharp contrast to the standard assumptions for a constant $\mathrm{SFR}$. We also found that, in contrast to $\epsilon_{\mathrm{FUV}, 8}, \tau_{50}$ is correlated with UV-optical color. This difference arises because while very recent star formation episodes not only makes galaxies bluer in the UV-optical color and dominate the UV luminosity, they also effectively erase the signatures from past SFR. Therefore, the
SFR at ages older than $\sim \tau_{50}$ simply adds noise to $\epsilon_{\mathrm{FUV}, 8}$. In effect, $\tau_{50}$ is a rough estimate of the timescale over which the $\mathrm{UV}$ is measuring the SFR, and it correlates with NUV $-r$ color.

\section{CONCLUSIONS}

In this study we have combined SFHs derived from resolved stellar CMDs with population synthesis modeling to predict the UV through NIR broadband SED of $~ 50$ dwarf galaxies drawn from the ANGST survey. We have compared these predicted SEDs to the observed SEDs on a galaxy-by-galaxy basis. We have also used these predicted SEDs to determine the effect of realistic star formation histories on the conversion between UV luminosity and SFR, and to derive characteristic timescales for the UV emission.

Summary of comparison to observations. The comparison of the predicted SEDs to the observed SEDs reveals excellent agreement in the optical portion of the spectrum ( $U$ through $i$ bands). This agreement lends support to the accuracy of the derived SFHs, though it is in some part expected given that the resolved stars used to derive the SFH typically contribute $\sim 40 \%$ of the total flux of the optical bands. In the GALEX UV bands we also find very good agreement between the predicted and observed luminosities when including a model for differential attenuation that does not include a significant scattering component.

Using Spitzer NIR photometry we have extended the comparison of population synthesis models to data to longer wavelength than in Melbourne et al. (2012). We find significant discrepancies between the predicted and observed integrated fluxes when using the Marigo et al. (2008) isochrones for TP-AGB stars. 

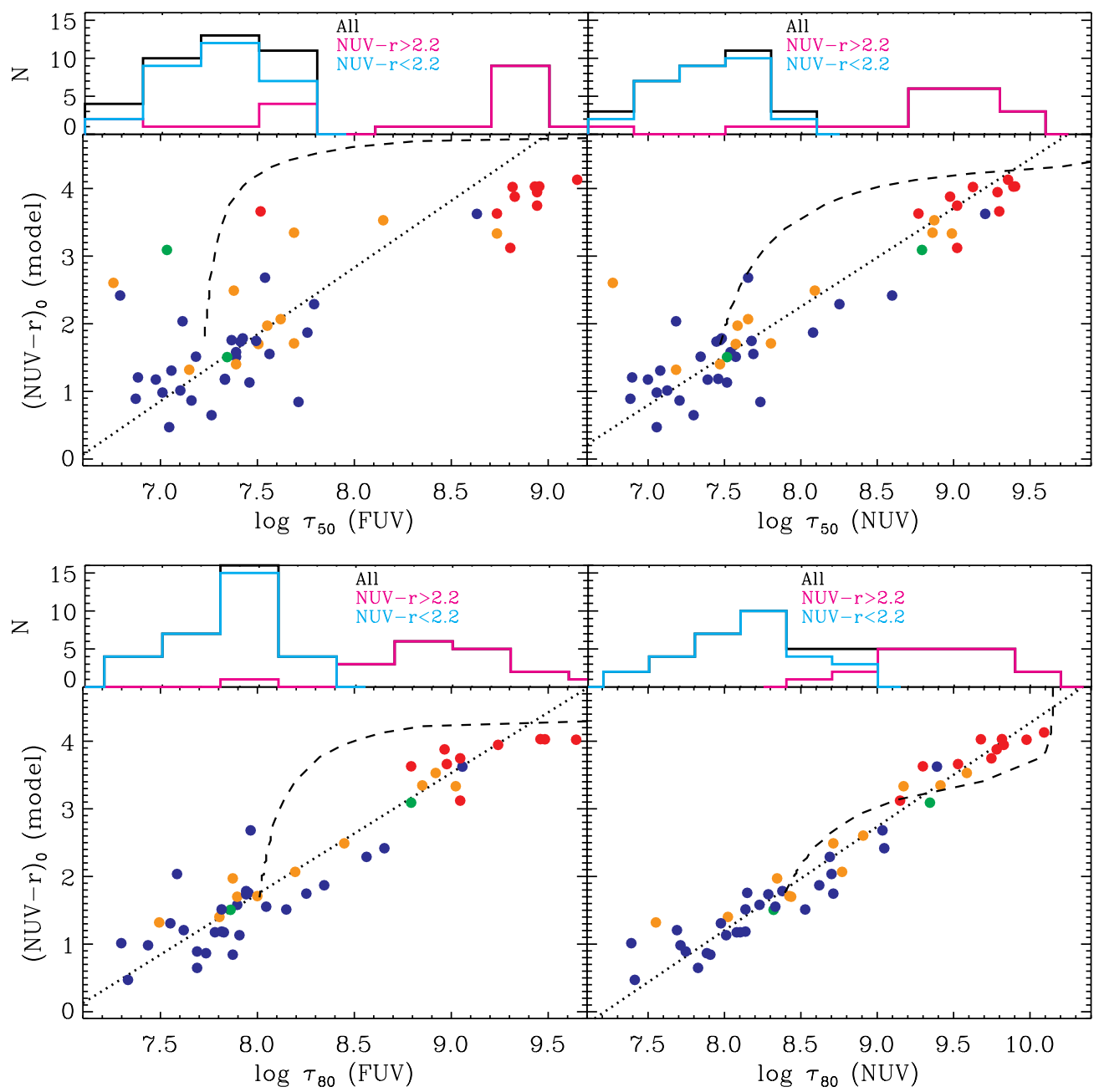

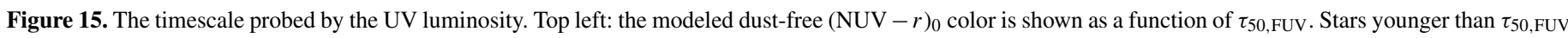
contribute 50\% of the modeled present day FUV luminosity. Points are color-coded by morphology: dSph (red), dTrans (orange), dSpiral (green), and dI (blue). The

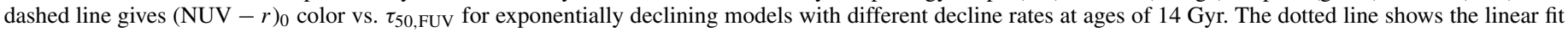

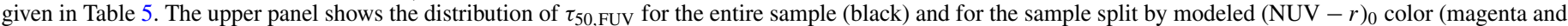
cyan). Top right: the same, but for $\tau_{50, \mathrm{NUV}}$. Bottom left: as for the top right, but (NUV $\left.-r\right)_{0}$ is plotted against $\tau_{80, \mathrm{FUV}}$. Stars younger than $\tau_{80, \mathrm{FUV}}$ contribute $80 \%$ of the modeled present day FUV flux. Bottom right: the same but for $\tau_{80, \mathrm{NUV}}$.

(A color version of this figure is available in the online journal.)

However, we are unable to distinguish the different stellar contributors at these wavelengths, limiting our conclusions as to the source of discrepancy. Nevertheless, we find that modifications to the TP-AGB isochrones as suggested in Conroy \& Gunn (2010) result in much better agreement between the observed and predicted luminosities at 3.6 and $4.5 \mu \mathrm{m}$.

We found that the differential extinction model that provides good matches to the optical CMD implies a larger than observed IR luminosity, under the assumption that all extinguished light is re-emitted in the IR. Including the effects of scattering can reduce this discrepancy, but at the cost of introducing a discrepancy in the FUV and NUV bands. We used the SFHs to calculate dust-free FUV - NUV colors and compared these both to UV colors predicted when including differential attenuation and to the observed UV colors, finding that variations in the derived SFHs of these galaxies are not sufficient to explain the average observed UV color and its scatter. The observed UV colors are consistent with differential extinction by dust.

Summary of model based results. Having gained confidence in the plausibility of the SFHs through comparison with observations-while keeping in mind the discrepancies-we have used the SFHs to determine the effect of realistic star formation histories on the conversion between UV luminosity and SFR. We found that differences in the star formation histories of these dwarf galaxies could cause variations in the conversion between FUV luminosity and the $\langle\mathrm{SFR}\rangle_{8}$ (the SFR averaged over $100 \mathrm{Myr}$ ) of an order of magnitude, with factor of two dispersion among individual galaxies in our sample. For our sample of dwarf galaxies SFH is likely the dominant cause of scatter in the ratio of observed UV luminosity to the SFR averaged over $100 \mathrm{Myr}$, above variations in dust attenuation or metallicity. We found that the variations in conversion from FUV luminosity to $\langle\mathrm{SFR}\rangle_{8}$ were only poorly correlated with broadband color, due to the lack of smoothness in the SFH.

Using the SFHs of the individual galaxies, we found that for significant numbers of our sample galaxies $50 \%$ of the FUV luminosity is produced by stars younger than $16 \mathrm{Myr}$, while for 10 of the sample galaxies $50 \%$ of the FUV luminosity was produced by stars older than $100 \mathrm{Myr}$. This timescale at which $50 \%$ of the luminosity is produced correlates with UVoptical color. We found also that the fraction of FUV luminosity 
produced by stars older than $100 \mathrm{Myr}$ ranges from less than $5 \%$ to $100 \%$.

Implications for SFR measurement. There is a factor of two dispersion in the ratio of modeled UV luminosity to $\langle\mathrm{SFR}\rangle_{8}$ that is due to SFH variations on short timescales. This large dispersion is in contrast to common conversions from FUV luminosity to SFR that adopt a constant SFH and predict zero dispersion. The dispersion that we find is not correlated with galaxy color, which suggests that it will be difficult to improve on the estimate of $\langle\mathrm{SFR}\rangle_{8}$ from UV luminosity or full SED modeling unless the SFH on short timescales can be otherwise constrained. Lee et al. (2010) have shown, using SFHs drawn from semi-analytic models, that modeling the SED of highredshift galaxies with an assumed SFH that does not match the true SFH can lead to biases and scatter in derived parameters. We have extended this result to low-redshift dwarf galaxies. Our factor of two dispersion is similar to the scatter in recovered versus input SFR that Lee et al. (2010) derive when using UV luminosity as a monochromatic SFR indicator (note that the biases and scatter in the recovered SFR of these simulated highredshift galaxies do not improve when fitting the entire SED).

Many current SED fitting procedures utilize large grids of models that include bursts of star-formation superimposed on a more smooth evolution (e.g, Brinchmann et al. 2004; Salim et al. 2007; Walcher et al. 2008; Moustakas et al. 2013). However, unless the age, duration, and amplitude of the bursts can be well constrained-which is exceedingly difficult even with optical spectroscopy-it will be impossible to choose the correct model and hence derive the correct SFR for an individual galaxy. It may be possible to properly incorporate the uncertainty on the derived SFR that is induced by shortterm SFH variations. However, even properly accounting for the uncertainty in the derived SFR (much less obtaining the correct SFR for individual galaxies) requires that the "burst" parameters (frequency, amplitude distribution, and durations) used in the library are representative of the population being fit (Walcher et al. 2011), whereas in practice they are rather arbitrarily defined (though see Pacifici et al. 2012 for a case where the library itself is based on semi-analytics models). The strong constraints on the SFH provided by CMDs of resolved stars have allowed us to accurately assess the uncertainty in derived SFR for a sample of nearby dwarf galaxies.

Applicability to other populations. It is important to consider the applicability of these results to different galaxy populations. The sample of galaxies we have considered is characterized by low stellar masses, low metallicities, low SFRs, and more stochastic SF. In more massive star forming galaxies, either locally or at high redshift, the recent SFH is likely to be more smooth than in the dwarfs of the present study. The scatter in the conversion between UV luminosity and SFR would then be smaller than we observe. However, when considering small parts of larger galaxies, for example to generate SFR maps, such SFH variations may be present and induce significant uncertainty in maps of SFR derived from UV luminosities (e.g., Bigiel et al. 2008; Leroy et al. 2012).

Based on observations made with the NASA/ESA Hubble Space Telescope, obtained at the Space Telescope Science Institute, which is operated by the Association of Universities for Research in Astronomy, Inc., under NASA contract NAS 5-26555. These observations are associated with programs 9771 , 9884, 10210, 10503, 10605, 10915, and 11986. We gratefully acknowledge NASA's support for construction, operation, and science analysis for the GALEX mission, developed in cooperation with the Centre National d'Etudes Spatiales of France and the Korean Ministry of Science and Technology. This work is based in part on observations made with the Spitzer Space Telescope, which is operated by the Jet Propulsion Laboratory, California Institute of Technology under a contract with NASA. This research has made extensive use of NASA's Astrophysics Data System Bibliographic Services. This research has made use of the NASA/IPAC Extragalactic Database, which is operated by JPL/Caltech, under contract with NASA.

Funding for SDSS-III has been provided by the Alfred P. Sloan Foundation, the Participating Institutions, the National Science Foundation, and the U.S. Department of Energy Office of Science. The SDSS-III Web site is http://www.sdss3.org/.

SDSS-III is managed by the Astrophysical Research Consortium for the Participating Institutions of the SDSS-III Collaboration including the University of Arizona, the Brazilian Participation Group, Brookhaven National Laboratory, University of Cambridge, Carnegie Mellon University, University of Florida, the French Participation Group, the German Participation Group, Harvard University, the Instituto de Astrofisica de Canarias, the Michigan State/Notre Dame/JINA Participation Group, Johns Hopkins University, Lawrence Berkeley National Laboratory, Max Planck Institute for Astrophysics, Max Planck Institute for Extraterrestrial Physics, New Mexico State University, New York University, Ohio State University, Pennsylvania State University, University of Portsmouth, Princeton University, the Spanish Participation Group, University of Tokyo, University of Utah, Vanderbilt University, University of Virginia, University of Washington, and Yale University.

\section{REFERENCES}

Aringer, B., Girardi, L., Nowotny, W., Marigo, P., \& Lederer, M. T. 2009, A\&A, 503,913

Arnouts, S., Walcher, C. J., Le Fèvre, O., et al. 2007, A\&A, 476, 137

Berg, D. A., Skillman, E. D., Marble, A. R., et al. 2012, ApJ, 754, 98

Bertelli, G., Bressan, A., Chiosi, C., Fagotto, F., \& Nasi, E. 1994, A\&AS, 106,275

Bigiel, F., Leroy, A., Walter, F., et al. 2008, AJ, 136, 2846

Blanton, M. R., Kazin, E., Muna, D., Weaver, B. A., \& Price-Whelan, A. 2011, AJ, 142, 31

Boquien, M., Buat, V., Boselli, A., et al. 2012, A\&A, 539, A145

Brinchmann, J., Charlot, S., White, S. D. M., et al. 2004, MNRAS, 351, 1151

Bruzual, A. G. 2007, in IAU Symp. 241, Stellar Populations as Building Blocks of Galaxies, ed. A. Vazdekis \& R. F. Peletier (Cambridge: Cambridge Univ. Press), 125

Bruzual, G., \& Charlot, S. 2003, MNRAS, 344, 1000

Calzetti, D., Armus, L., Bohlin, R. C., et al. 2000, ApJ, 533, 682

Cardelli, J. A., Clayton, G. C., \& Mathis, J. S. 1989, ApJ, 345, 245

Cerviño, M., \& Luridiana, V. 2009, in New Quests in Stellar Astrophysics. II. Ultraviolet Properties of Evolved Stellar Populations, ed. M. Chávez Dagostino, E. Bertone, D. Rosa Gonzalez, \& L. H. Rodriguez-Merino (Dordrecht: Springer), 293

Cerviño, M., \& Valls-Gabaud, D. 2003, MNRAS, 338, 481

Charlot, S., Worthey, G., \& Bressan, A. 1996, ApJ, 457, 625

Conroy, C., \& Gunn, J. E. 2010, ApJ, 712, 833

Conroy, C., Gunn, J. E., \& White, M. 2009, ApJ, 699, 486

Curtis-Lake, E., McLure, R. J., Dunlop, J. S., et al. 2013, MNRAS, 429, 302

da Cunha, E., Charlot, S., \& Elbaz, D. 2008, MNRAS, 388, 1595

Dalcanton, J. J., Williams, B. F., Lang, D., et al. 2012, ApJS, 200, 18

Dalcanton, J. J., Williams, B. F., Seth, A. C., et al. 2009, ApJS, 183, 67

Dale, D. A., Cohen, S. A., Johnson, L. C., et al. 2009, ApJ, 703, 517

Dale, D. A., Gil de Paz, A., Gordon, K. D., et al. 2007, ApJ, 655, 863

da Silva, R. L., Fumagalli, M., \& Krumholz, M. 2012, ApJ, 745, 145

de Vaucouleurs, G., de Vaucouleurs, A., Corwin, H. G., Jr., et al. 1991, in

Third Reference Catalogue of Bright Galaxies. Volume I: Explanations and references. Volume II: Data for galaxies between $0^{h}$ and $12^{h}$. Volume III: Data for galaxies between $12^{h}$ and $24^{h}$ (New York: Springer)

Dohm-Palmer, R. C., Skillman, E. D., Mateo, M., et al. 2002, AJ, 123, 813 
Dolphin, A. E. 2002, MNRAS, 332, 91

Dolphin, A. E. 2012, ApJ, 751, 60

Dolphin, A. E., Saha, A., Skillman, E. D., et al. 2003, AJ, 126, 187

Eldridge, J. J. 2012, MNRAS, 422, 794

Eskew, M., \& Zaritsky, D. 2011, AJ, 141, 69

Fazio, G. G., Hora, J. L., Allen, L. E., et al. 2004, ApJS, 154, 10

Fumagalli, M., da Silva, R. L., \& Krumholz, M. R. 2011, ApJL, 741, L26

Gil de Paz, A., Boissier, S., Madore, B. F., et al. 2007, ApJS, 173, 185

Girardi, L., Bressan, A., Bertelli, G., \& Chiosi, C. 2000, A\&AS, 141, 371

Girardi, L., Williams, B. F., Gilbert, K. M., et al. 2010, ApJ, 724, 1030

Gogarten, S. M., Dalcanton, J. J., Williams, B. F., et al. 2009, ApJ, 691, 115

Grocholski, A. J., van der Marel, R. P., Aloisi, A., et al. 2012, AJ, 143, 117

Hao, C.-N., Kennicutt, R. C., Johnson, B. D., et al. 2011, ApJ, 741, 124

Hidalgo, S. L., Aparicio, A., Skillman, E., et al. 2011, ApJ, 730, 14

Hogg, D. W., Tremonti, C. A., Blanton, M. R., et al. 2005, ApJ, 624, 162

Hopkins, P. F., Hernquist, L., Cox, T. J., et al. 2006, ApJS, 163, 1

Huang, M.-L., Kauffmann, G., Chen, Y.-M., et al. 2013, MNRAS, 431, 2622

Johnson, B. D., Schiminovich, D., Seibert, M., et al. 2007a, ApJS, 173, 377

Johnson, B. D., Schiminovich, D., Seibert, M., et al. 2007b, ApJS, 173, 392

Karachentsev, I. D., Karachentseva, V. E., Huchtmeier, W. K., \& Makarov, D. I. 2004, AJ, 127, 2031

Kauffmann, G., Heckman, T. M., White, S. D. M., et al. 2003, MNRAS, 341,33

Kennicutt, R. C. 1998, ARA\&A, 36, 189

Kennicutt, R. C., Jr., \& Evans, N. J., II 2012, ARA\&A, 50, 531

Kong, X., Charlot, S., Brinchmann, J., \& Fall, S. M. 2004, MNRAS, 349, 769

Kriek, M., Labbé, I., Conroy, C., et al. 2010, ApJL, 722, L64

Lançon, A., \& Mouhcine, M. 2002, A\&A, 393, 167

Lee, H., Skillman, E. D., Cannon, J. M., et al. 2006, ApJ, 647, 970

Lee, J. C., Gil de Paz, A., Kennicutt, R. C., Jr., et al. 2011, ApJS, 192, 6

Lee, J. C., Gil de Paz, A., Tremonti, C., et al. 2009, ApJ, 706, 599

Lee, S.-K., Ferguson, H. C., Somerville, R. S., Wiklind, T., \& Giavalisco, M. 2010, ApJ, 725, 1644

Leroy, A. K., Bigiel, F., de Blok, W. J. G., et al. 2012, AJ, 144, 3

Madau, P., Pozzetti, L., \& Dickinson, M. 1998, ApJ, 498, 106

Madden, S. C. 2000, NewAR, 44, 249

Mancone, C. L., \& Gonzalez, A. H. 2012, PASP, 124, 606

Maraston, C. 2005, MNRAS, 362, 799

Maraston, C., Pforr, J., Henriques, B. M., et al. 2012, arXiv:1207.6114

Marble, A. R., Engelbracht, C. W., van Zee, L., et al. 2010, ApJ, 715, 506

Marigo, P., Girardi, L., Bressan, A., et al. 2008, A\&A, 482, 883

Martin, D. C., Fanson, J., Schiminovich, D., et al. 2005, ApJL, 619, L1

McIntosh, D. H., Bell, E. F., Weinberg, M. D., \& Katz, N. 2006, MNRAS, 373, 1321

Meidt, S. E., Schinnerer, E., Knapen, J. H., et al. 2012, ApJ, 744, 17

Melbourne, J., Williams, B. F., Dalcanton, J. J., et al. 2012, ApJ, 748, 47
Mentuch Cooper, E., Wilson, C. D., Foyle, K., et al. 2012, ApJ, 755, 165

Meurer, G. R., Heckman, T. M., \& Calzetti, D. 1999, ApJ, 521, 64

Moustakas, J., Zaritsky, D., Brown, M., et al. 2013, ApJ, 767, 50

Noll, S., Burgarella, D., Giovannoli, E., et al. 2009, A\&A, 507, 1793

Pacifici, C., Charlot, S., Blaizot, J., \& Brinchmann, J. 2012, MNRAS, 421, 2002

Pei, Y. C. 1992, ApJ, 395, 130

Pforr, J., Maraston, C., \& Tonini, C. 2012, MNRAS, 422, 3285

Rauch, T. 2002, in Rev. Mex. Astron. Astrofis. Conf. Ser., Vol. 12, Ionized Gaseous Nebulae, A Conference to Celebrate the 60th Birthdays of Silvia Torres-Peimbert and Manuel Peimbert, ed. W. J. Henney, J. Franco, \& M. Martos, 150

Reddy, N., Dickinson, M., Elbaz, D., et al. 2012, ApJ, 744, 154

Salim, S., Charlot, S., Rich, R. M., et al. 2005, ApJL, 619, L39

Salim, S., Rich, R. M., Charlot, S., et al. 2007, ApJS, 173, 267

Sanna, N., Bono, G., Stetson, P. B., et al. 2009, ApJL, 699, L84

Schaerer, D., \& de Barros, S. 2010, A\&A, 515, A73

Schiminovich, D., Wyder, T. K., Martin, D. C., et al. 2007, ApJS, 173, 315

Schlegel, D. J., Finkbeiner, D. P., \& Davis, M. 1998, ApJ, 500, 525

Schneider, D. P., Gunn, J. E., \& Hoessel, J. G. 1983, ApJ, 264, 337

Seibert, M., Martin, D. C., Heckman, T. M., et al. 2005, ApJL, 619, L55

Silva, L., Granato, G. L., Bressan, A., \& Danese, L. 1998, ApJ, 509, 103

Smit, R., Bouwens, R. J., Franx, M., et al. 2012, ApJ, 756, 14

Smith, L. J., Norris, R. P. F., \& Crowther, P. A. 2002, MNRAS, 337, 1309

Srinivasan, S., Sargent, B. A., \& Meixner, M. 2011, A\&A, 532, A54

Stinson, G. S., Dalcanton, J. J., Quinn, T., Kaufmann, T., \& Wadsley, J. 2007, ApJ, 667, 170

Stringer, M., Cole, S., Frenk, C. S., \& Stark, D. P. 2011, MNRAS, 414, 1927

Tinsley, B. M. 1968, ApJ, 151, 547

Tosi, M., Greggio, L., \& Focardi, P. 1989, Ap\&SS, 156, 295

Vassiliadis, E., \& Wood, P. R. 1994, ApJS, 92, 125

Walcher, C. J., Lamareille, F., Vergani, D., et al. 2008, A\&A, 491, 713

Walcher, J., Groves, B., Budavári, T., \& Dale, D. 2011, Ap\&SS, 331, 1

Weisz, D. R., Dalcanton, J. J., Williams, B. F., et al. 2011, ApJ, 739, 5

Werner, M. W., Roellig, T. L., Low, F. J., et al. 2004, ApJS, 154, 1

Westera, P., Lejeune, T., Buser, R., Cuisinier, F., \& Bruzual, G. 2002, A\&A, 381,524

Whitaker, K. E., van Dokkum, P. G., Brammer, G., \& Franx, M. 2012, ApJL, 754, L29

Wild, V., Walcher, C. J., Johansson, P. H., et al. 2009, MNRAS, 395, 144

Wilkins, S. M., Gonzalez-Perez, V., Lacey, C. G., \& Baugh, C. M. 2012, MNRAS, 427, 1490

Witt, A. N., \& Gordon, K. D. 2000, ApJ, 528, 799

Wu, R., Hogg, D. W., \& Moustakas, J. 2011, ApJ, 730, 111

Wyder, T. K. 2001, AJ, 122, 2490

Zibetti, S., Gallazzi, A., Charlot, S., Pasquali, A., \& Pierini, D. 2013, MNRAS, 428,1479 